\title{
Energie und Umwelt: Was kann Technikfolgenabschätzung leisten?
}

\section{$1 \quad$ Problemstellung}

Die Nutzung neuer Energietechniken und die Verfügbarmachung neuer Energiequellen waren wesentliche Voraussetzungen für die Entwicklung unserer modernen Industriegesellschaft. Die zunehmende Nutzung von Energie hat aber auch unerwünschte Nebeneffekte und nicht intendierte Wirkungen deutlicher hervortreten lassen. Die Umwelt- und Klimawirkungen der Energienutzung, die Risiken einzelner Energietechniken, die gesamtwirtschaftlichen Konsequenzen des Verzichts auf eine Energieoption, aber auch die sozialen und gesellschaftlichen Wirkungen von Energietechniken stehen seit fast zwei Jahrzehnten im Zentrum der energie- und gesellschaftspolitischen Diskussion und verdeutlichen den hier bestehenden Bedarf fundierter Technikfolgenforschung und Technikfolgenabschätzung als Grundlage einer rationalen Technikbewertung.

Einige wenige Beispiele sollen die Bedeutung der Energieumwandlung bei der Entstehung von Umweltbelastungen verdeutlichen, wobei auch der Sektor Verkehr der Energieumwandlung zugerechnet wird. Bild 1 zeigt den Anteil der Energieumwandlung einschließlich des Verkehrs an den gesamten Luftschadstoffemissionen einiger wichtiger Schadstoffe in der Bundesrepublik. Energieumwandlungsprozesse verursachten 1990 $99 \%$ der $\mathrm{NO}_{\mathrm{x}}$-Emissionen, $90 \%$ der $\mathrm{SO}_{2}$-Emissionen, $92,5 \%$ der $\mathrm{CO}$ Emissionen und ca. $54 \%$ (1989) der anthropogenen VOC-Emissionen in der Bundesrepublik Deutschland und tragen damit entscheidend zu den Problemen, die die Luftverschmutzung mit sich bringt, bei. 


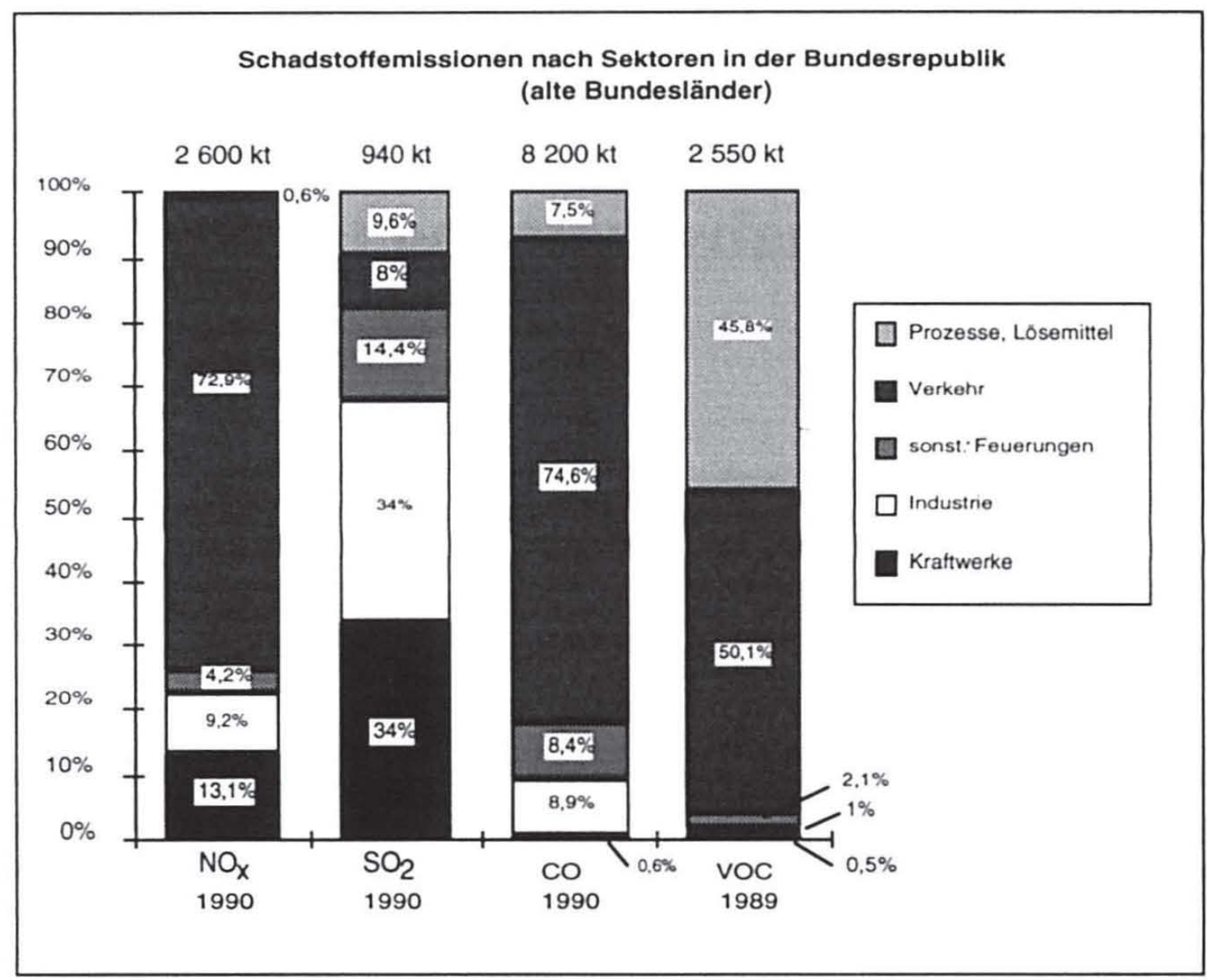

Bild 1 Schadstoffemissionen nach Sektoren in der Bundesrepublik 1990 bzw. 1989 (alte Bundesländer) /1/

Eines der derzeit meist diskutierten Umweltprobleme ist der Treibhauseffekt. Bild 2 zeigt, daß der Anteil der Energienutzung am anthropogen verursachten Treibhauseffekt ca. $50 \%$ beträgt. Speziell der Bereich Verkehr verursacht noch eine Reihe weiterer Risiken und Umweltprobleme. So wurden in der Bundesrepublik (gesamt) 199210627 Personen bei Straßenverkehrsunfällen getötet /8/. Zusätzlich wurden 1992130000 Personen schwer und 386000 Personen leicht verletzt.

Nach /7/ fühlen sich etwa $23 \%$ der Bevölkerung der alten Bundesländer, das sind ca. 14 Mio. Menschen, durch Straßenverkehrslärm stark belästigt. $12 \%$ der Bevölkerung (7 Mio. Menschen) sind tagsüber einem Geräuschpegel von $65 \mathrm{~dB}(\mathrm{~A})$ und mehr ausgesetzt, der Gesundheitsschäden hervorrufen kann. Nachts müssen etwa $28 \%$ der Bevölkerung (17 Mio.) Geräuschpegel von $50 \mathrm{~dB}(\mathrm{~A})$ und mehr, die zu Schlafstörungen führen können, erdulden $/ 4 /$. 


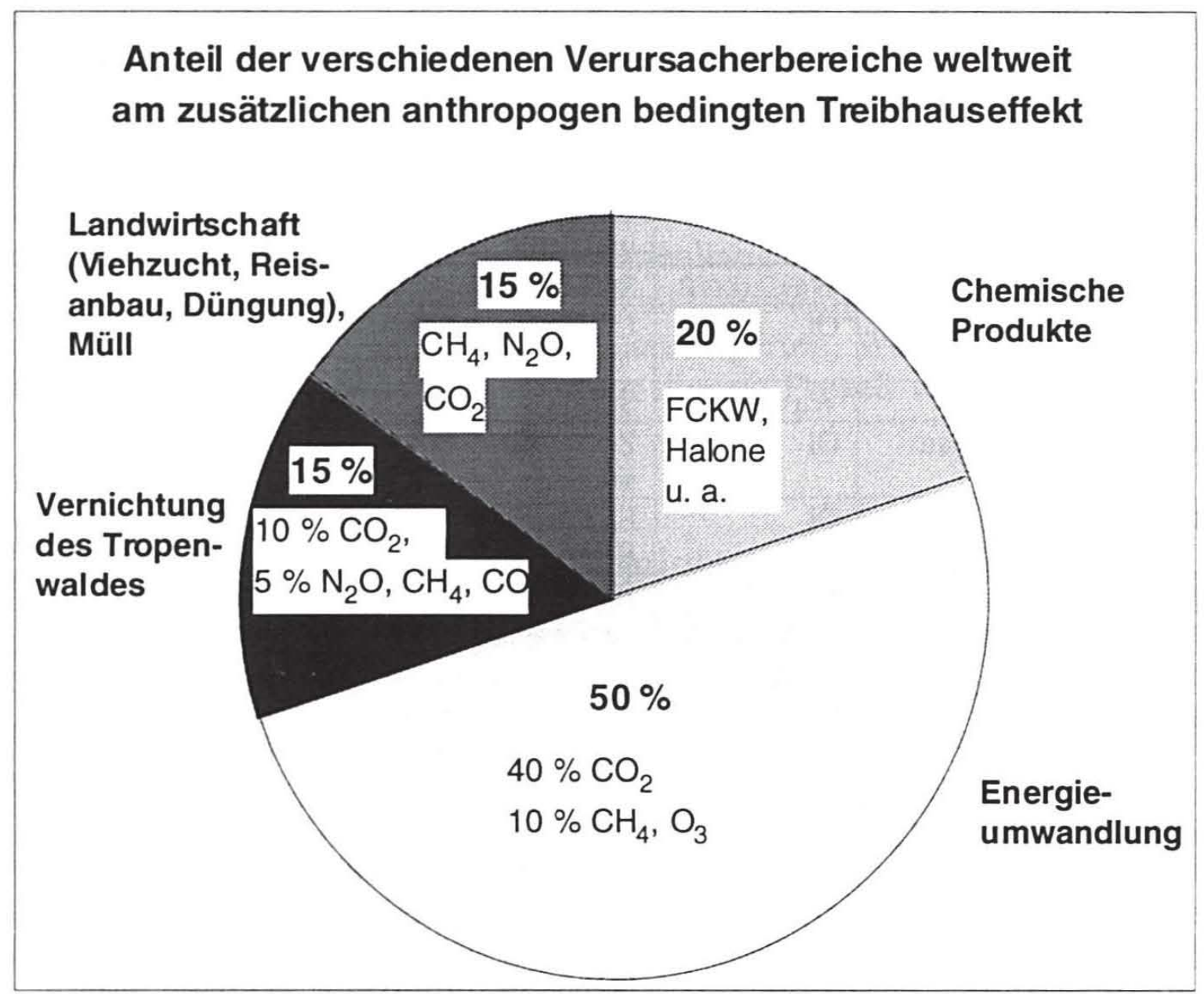

Bild 2 Anteil der verschiedenen Verursacherbereiche weltweit am zusätzlichen anthropogen bedingten Treibhauseffekt /2/

Als weitere, vom Verkehr verursachte Umweltauswirkung ist der Flächenverbrauch zu nennen. Derzeit werden sowohl in den alten Bundesländern insgesamt als auch in Baden-Württemberg $5 \%$ der Gesamtfläche als Verkehrsfläche genutzt; Siedlungs- und Verkehrsflächen zusammen machen bereits ca. $12 \%$ der Gesamtfläche aus. Auch außerhalb des Sektors Verkehr verursacht die Energieversorgung Beeinträchtigungen durch Flächenverbrauch und Risiken für die menschliche Gesundheit. So nimmt die Braunkohleförderung in Ost- und Westdeutschland große Flächen in Anspruch. Sollten in Zukunft erneuerbare Energieträger einen größeren Anteil an der Energieversorgung übernehmen, so würde dies zu weiteren ganz erheblichen Flächenverbräuchen für die Energieversorgung führen. $\mathrm{Da}$ mit der Energieversorgung auch erhebliche Risiken verbunden sein können, zeigt Tab. 1. Hier sind im letzten Jahrzehnt geschehene schwere Unfälle, die im Zusammenhang mit der Energieversorgung stehen, aufgeführt. 


\begin{tabular}{|c|c|c|c|c|}
\hline Jahr & Ort/Land & $\begin{array}{l}\text { Energie- } \\
\text { art }\end{array}$ & Unfallart & Folgen \\
\hline 1980 & Nordsee & Öı & $\begin{array}{l}\text { Kentern der Ölplattform } \\
\text { "Alexander Kielland" }\end{array}$ & 123 Tote \\
\hline 1980 & Türkei & Flüssiggas & $\begin{array}{l}\text { Explosion einer Flasche } \\
\text { und Brand }\end{array}$ & 97 Tote \\
\hline 1980 & Indien & Wasserkraft & Staudammbruch & rd. 2000 Tote \\
\hline 1982 & Neufundland & Öı & $\begin{array}{l}\text { Untergang der Bohrinsel } \\
\text { "Ocean Ranger" }\end{array}$ & 84 Tote \\
\hline 1982 & Liberia & Wasserkraft & Staudammbruch & rd. 200 Tote \\
\hline 1982 & Afghanistan & Öl & $\begin{array}{l}\text { Zusammensto B von Tank- } \\
\text { wagen in Tunnel }\end{array}$ & rd. 1100 Tote \\
\hline 1982 & Venezuela & ÖI & $\begin{array}{l}\text { Explosion von Öltanks in } \\
\text { Kraftwerk }\end{array}$ & 145 Tote \\
\hline 1982 & China & Kohle, Müll & Haldeneinsturz & 284 Tote \\
\hline 1983 & Kolumbien & Wasserkraft & Staudammbruch & rd. 150 Tote \\
\hline 1984 & China & Kohle & Gruben-Explosion & rd. 100 Tote \\
\hline 1984 & Mexiko & Erdgas & $\begin{array}{l}\text { Explosion einer Erdgas- } \\
\text { Verarbeitungsanlage und } \\
\text { Brand }\end{array}$ & 452 Tote \\
\hline 1984 & Taiwan & Kohle & Grubenbrand & 121 Tote \\
\hline 1984 & Brasilien & ÖI & $\begin{array}{l}\text { Explosion einer Pipeline } \\
\text { und Brand }\end{array}$ & über 500 Tote \\
\hline 1986 & UdSSR & Kernenergie & $\begin{array}{l}\text { Leistungs-Explosion und } \\
\text { Brand eines RBMK- } \\
\text { Reaktors }\end{array}$ & $\begin{array}{l}31 \text { Soforttote, } \\
\text { erhebl. Lang- } \\
\text { zeitfolgen noch } \\
\text { nicht quantifi- } \\
\text { zierbar }\end{array}$ \\
\hline 1988 & Schottland & Öl & $\begin{array}{l}\text { Explosion der Förder- } \\
\text { Plattform "Piper Alpha" }\end{array}$ & 166 Tote \\
\hline 1989 & $\begin{array}{l}\text { Tscheljabinsk } \\
\text { (Ural/UdSSR) }\end{array}$ & Flüssiggas & $\begin{array}{l}\text { Explosion einer Leitung } \\
\text { während des Vorbeifah- } \\
\text { rens von } 2 \text { Zugen }\end{array}$ & 607 Tote \\
\hline 1989 & $\begin{array}{l}\text { Prince-William- } \\
\text { Sund (Alaska) } \\
\end{array}$ & Öl & Havarie eines Tankers & Ólpest \\
\hline
\end{tabular}

Tab. 1 Schwere Unfälle im Energiesektor seit 1980 (ab etwa 100 Tote)

Da der Nutzen und die Risiken bzw. unerwünschten Nebeneffekte der uns heute zur Verfügung stehenden Energieträger und Energiesysteme ebenso wie die der sich im Entwicklungsstadium befindlichen neuen Energieträger und Energiesysteme sehr unterschiedlich sind, kommt fundierten und umfassend angelegten Technikfolgenabschätzungen als Grundlage einer Technikbewertung zur Realisierung eines Energiesystems, das ausreichende Energiemengen für eine wachsende Weltbevölkerung bereitstellt und die Inanspruchnahme der knappen Ressourcen 
Umwelt und Natur so begrenzt, daß die natürlichen Lebensgrundlagen auf Dauer erhalten und die Klimaveränderungen auf ein noch tolerierbares $\mathrm{Ma} \beta$ begrenzt werden, eine wichtige Aufgabe zu.

Bevor im folgenden exemplarisch auf einige Technikfolgenabschätzungen im Energiebereich näher eingegangen wird, soll noch ein Aspekt angesprochen werden, der im Zusammenhang mit der Abgrenzung von Technikfolgenabschätzung und Technikbewertung sowie der Rolle und Aufgabe von Wissenschaft, technischen Experten, Wirtschaft, Politik und anderen gesellschaftlichen Gruppen in diesem Prozeß wichtig erscheint.

\section{Zum Verständnis wissenschaftlicher Technikfolgenabschätzung}

Gerade in der Diskussion über die Zukunft der Energieversorgung und die Bewältigung der energiebedingten Umweltprobleme ist in den letzten Jahren eine bedauerliche Vermischung von Politik und Wissenschaft, von Werturteil und wissenschaftlich gesicherter Erkenntnis eingetreten. Dabei sind die klaren Verantwortungsbereiche der Beteiligten verwischt worden. Dies hat der Wissenschaft und den Experten geschadet, der Politik nicht geholfen und die Bürger zunehmend verunsichert.

Grundsatzentscheidungen über die zukünftige Ausgestaltung unserer Energieversorgung sind letztlich keine technisch und wissenschaftlich ableitbaren Entscheidungen. Bei dem dabei notwendigen Abwägen von Nutzen und Risiken geht es um mehr als um technisch-ökonomische oder naturwissenschaftliche Sachverhalte. Die hiermit angedeutete Differenzierung zwischen Fakten und mehr oder weniger gesicherten Erkenntnis auf der einen Seite und politischem Werturteil auf der anderen Seite umreißt in unserem demokratischen Gemeinwesen den Aufgabenund Zuständigkeitsbereich von Wissenschaft und Technikexperten gegenüber dem der Politik und anderen gesellschaftlichen Gruppen und grenzt sie gegeneinander ab. Wissenschaft und wissenschaftliche Politikberatung, also auch Technikfolgenabschätzung (TA), haben die Aufgabe wissenschaftliche Wahrheiten, also zutreffende Aussagen über die Wirklichkeit bereitzustellen. Politik fällt Wertentscheidungen. Im Unterschied zur wissenschaftlichen Wahrheit gibt es keine politische Wahrheit, sondern nur ein politisches Werturteil.

Die Wissenschaft und die technischen Experten müssen der Politik Grenzlinien mitteilen, bis zu denen wissenschaftliche Wahrheiten und Er- 
kenntnisse gewonnen werden können, denn darüber besteht keine Freiheit der Werturteile. Der zweite Hauptsatz der Thermodynamik läßt sich nicht durch Parlamentsentscheid außer Kraft setzen. Andererseits gilt aber auch, daß die politische Schlußfolgerung aus dem, was wissenschaftlich gesichert ist, in einem demokratischen Gemeinwesen nicht Sache der Wissenschaft oder der Experten ist. Dies ist von einzelnen Experten und Wissenschaftlern in der Vergangenheit nicht beachtet worden, sie haben unter dem Mantel der Wissenschaft Wertentscheidungen gerade in der Öffentlichkeit vertreten. In bezug auf politische Werturteile kann aber der Wissenschaftler oder Experte keine besondere moralische und sachliche Autorität für sich in Anspruch nehmen.

Nun ist eine ganz strikte Trennung zwischen Fakten und Werturteil bei komplexen Technikfolgenabschätzungen nur eingeschränkt möglich, weil auch in Annahmen, Vereinfachungen, Berücksichtigung und Nichtberücksichtigung von Relationen usw. Bewertungen einfließen. Es ist in diesen Fällen sicherzustellen, daß die impliziten Wertentscheidungen das Ergebnis um mindestens eine Größenordnung weniger beeinflussen als die in die nachfolgende Bewertung einfließenden Werturteile. Ist dies nicht möglich, so kann und soll durch Parametervariation der Einfluß des implizit einfließenden Werturteils auf das Ergebnis gezeigt werden. Jedenfalls ist eine weitgehende Trennung zwischen Fakten und Werturteil anzustreben, weil die bedenkenlose Vermischung ohne Zweifel zum Elend der Experten geführt und die Wissenschaft in Mißkredit gebracht hat.

Eine striktere Trennung von Fakten und wissenschaftlicher Erkenntnis sowie ihrer politischen Bewertung, also die Trennung von Technikfolgenabschätzung und Technikbewertung, ist eine wesentliche Vorbedingung, um den gesellschaftlichen Diskurs über den Einsatz von Technik zur Bewältigung der vor uns liegenden Herausforderungen fruchtbarer zu machen. Wissenschaftler und Experten müssen im Rahmen der Politikberatung wissenschaftlich anspruchsvoller, aber politisch bescheidener werden. Es muß solchen Methoden der Technikfolgenabschätzung und der Politikberatung der Vorzug gegeben werden, die einer wissenschaftlichen Analyse breiten Raum geben und es erlauben, die wissenschaftlich zwingenden Schlußfolgerungen von denen zu trennen, die der politischen bzw. gesellschaftlichen Entscheidung vorbehalten bleiben müssen.

Dies scheint eine wesentliche Vorbedingung zu sein, damit die Rationalität den ihr gebührenden Platz im Rahmen der vor uns liegenden weitreichenden Entscheidungen zur Bewältigung der Energie- und Umweltprobleme zurückgewinnen kann. 


\section{Beispiele von TA-Studien im Energiebereich}

Im folgenden sollen beispielhaft einige wenige Studien aus den verschiedenen Kategorien von TA-Studien vorgestellt werden. Folgende Kategorisierung der TA-Studien im Bereich Energie bietet sich an:

- Technikinduzierte Technikfolgenabschätzung: Ermittlung der Potentiale des Einsatzes sowie der Folgen des Einsatzes einer Technik oder Technikfamilie (z. B. Wasserstoff als Sekundärenergieträger, Windenergiekonverter, Kernfusion, Photovoltaik).

- Probleminduzierte Technikfolgenabschätzung: Erarbeitung von Strategien zur Lösung akuter oder zukünftiger zu erwartender Probleme. Dabei dient die bedarfsinduzierte Technikfolgenabschätzung der Ermittlung von Möglichkeiten zur Deckung eines zukünftigen Bedarfs an Energiedienstleistungen und der Folgen (Vor- und Nachteile) alternativer Wege der Bedarfsdeckung; die folgeninduzierte Technikfolgenabschätzung ermittelt Möglichkeiten zur Verringerung von negativen Folgen bzw. unerwünschten Effekten, die mit der Energieumwandlung verbunden sind (z. B. Klimaänderung, hohe Ozonkonzentration) und versucht eine möglichst weitgehende Quantifizierung der Vor- und Nachteile dieser Möglichkeiten.

Das methodische Vorgehen und einige exemplarische Ergebnisse solcher Studien sollen im folgenden an jeweils einem Beispiel von konkret durchgeführten TA-Studien für jede TA-Kategorie aufgezeigt werden. In den beschriebenen Beispielen wird kein Anspruch auf eine vollständige Behandlung aller Aspekte einer Technikfolgenabschätzung erhoben, vielmehr soll die prinzipielle Vorgehensweise deutlich werden.

\subsection{Technikinduzierte Technikfolgenabschätzung: Bedingungen und Folgen von Aufbaustrategien für eine solare Wasserstoffwirtschaft}

Unter dem Eindruck, daß die Folgen moderner Technik in ihren Nutzenund Risikodimensionen an Bedeutung gewinnen und daß die Bevölkerung auch die negativen Folgen der Technikanwendung zunehmend wahrnimmt, wurden im Deutschen Bundestag Forderungen nach einer 
Verbesserung des Informations- und Wissenstandes über Techniken, für die in Zukunft ein Entscheidungsbedarf besteht, laut. Daraufhin wurde 1985 die Enquête-Kommission Einschätzung und Bewertung von Technikfolgen, Gestaltung von Rahmenbedingungen der technischen Entwicklung ins Leben gerufen, die u. a. Arbeiten auf den Gebieten Expertensysteme, Nachwachsende Rohstoffe und Alternative landwirtschaftliche Produktionsweisen begonnen hat. In der nachfolgenden 11. Wahlperiode des Deutschen Bundestages wurde dann 1987 erneut eine EnquêteKommission Einschätzung und Bewertung von Technikfolgenabschätzung, Gestaltung von Rahmenbedingungen der technischen Entwicklung eingesetzt, die die Arbeit der vorhergehenden Kommission fortführte und zusätzlich das Arbeitsfeld Bedingungen und Folgen von Aufbaustrategien für eine solare Wasserstoffwirtschaft aufgriff. Im Rahmen dieses Arbeitsfeldes sollten die folgenden Fragen untersucht werden:

a Welche Entwicklungslinien führen zum Einsatz von solarem Wasserstoff?

- Unter welchen Bedingungen kann der solare Wasserstoff einen nennenswerten Beitrag zur Energieversorgung leisten?

J Welche Folgen hat die Nutzung solaren Wasserstoffs?

Zur Beantwortung dieser Fragen wurde folgendes Vorgehen gewählt /5/: Zunächst wurde eine Datenbasis zu technischen, ökonomischen und umweltrelevanten Parametern von Schlüsseltechnologien für den Aufbau einer solaren Wasserstoffwirtschaft erstellt. Ausgehend von dieser Datenbasis und festgelegten Rahmenbedingungen wurden dann verschiedene konsistente Szenarien (sog. Aufbaustrategien) mit unterschiedlichen, aber immer erheblichen Anteilen an solarem Wasserstoff bis 2050 für die Bundesrepublik (alte Bundesländer) entwickelt. Diese Strategien wurden mit Fachleuten und den Mitgliedern der Kommission diskutiert. Anschließend wurden technische, ökonomische, soziale und politische Folgen sowie Realisierungsbedingungen der Szenarien analysiert. Entscheidend für die Aussagekraft der Ergebnisse sind natürlich die für die Szenarien gewählten Randbedingungen und Annahmen. Insbesondere wurden folgende Annahmen getroffen:

(1) Die $\mathrm{CO}_{2}$-Emissionen werden zur Verringerung der Folgen des Treibhauseffekts bis 2005 um $25 \%$ und bis 2050 um $75 \%$ reduziert. Diese Vorgabe ist eine entscheidende Vorbedingung für den Einsatz solaren Wasserstoffs, weil damit die Begrenzung des Einsatzes fossiler Energieträger begründet werden kann.

(2) Um den unterschiedlichen Ansichten über die Vertretbarkeit der Kernenergie Rechnung zu tragen, werden zwei Hauptpfade gebildet. Im 
Hauptpfad I erfolgt ein Abschalten der Kernenergie bis 2005, im Hauptpfad II erfolgt ein deutlicher, aber ohne weitere Begründung begrenzter Ausbau der Kernenergie auf etwa das Doppelte der heutigen Kapazität bis 2050 .

(3) Bei allen Pfaden wird die gleiche demographische Entwicklung und das gleiche Wirtschaftswachstum unterstellt.

(4) Der Primärenergieverbrauch sinkt bei Hauptpfad I ab 1988 um $26 \%$ bis 2005 und um $44 \%$ bis 2050. Bei Hauptpfad II wird ein Absinken um $7 \%$ bis 2005 und um $23 \%$ bis 2050 unterstellt.

(5) Der Primärenergieverbrauch fossiler Energieträger sinkt bei allen Aufbaupfaden von 2709 TWh 1988 auf 651 TWh 2050.

(6) Nach und nach werden die folgenden technischen Potentiale an erneuerbaren Energieträgern im Inland dezentral genutzt:

- solarthermische Kollektoren: 127 TWh (th)/a;

口 Wärmepumpen: $31 \mathrm{TWh}$ (th)/a;

口 Biomasse und Müll-Wärme: 95 TWh (th)/a;

口 Biomasse und Müll-Strom: 25 TWh (el)/a;

Wasserkraft: 30 TWh (el)/a;

Windenergie: $30 \mathrm{TWh}(\mathrm{el}) / \mathrm{a}$;

口 Photovoltaik (Dächer/Fassaden): 50 TWh (el)/a.

(7) Zusätzlicher Bedarf wird durch Photovoltaikanlagen, insbesondere im Ausland, und Wasserkraftnutzung im Ausland, gedeckt. Dabei wird Strom z. T. in Wasserstoff umgewandelt und als Wasserstoff transportiert und gespeichert.

(8) Der Anteil erneuerbarer Energieträger am Primärenergieverbrauch beträgt 2050 im Hauptpfad I $63 \%$, im Hauptpfad II $39 \%$. Solarer Wasserstoff ist 2050 beim Hauptpfad I zu ca. $25 \%$, bei Hauptpfad II zu ca. $12 \%$ am Primärenergieverbrauch beteiligt. Bild 3 zeigt den Primärenergieverbrauch der beiden Hauptpfade bis 2050 . 


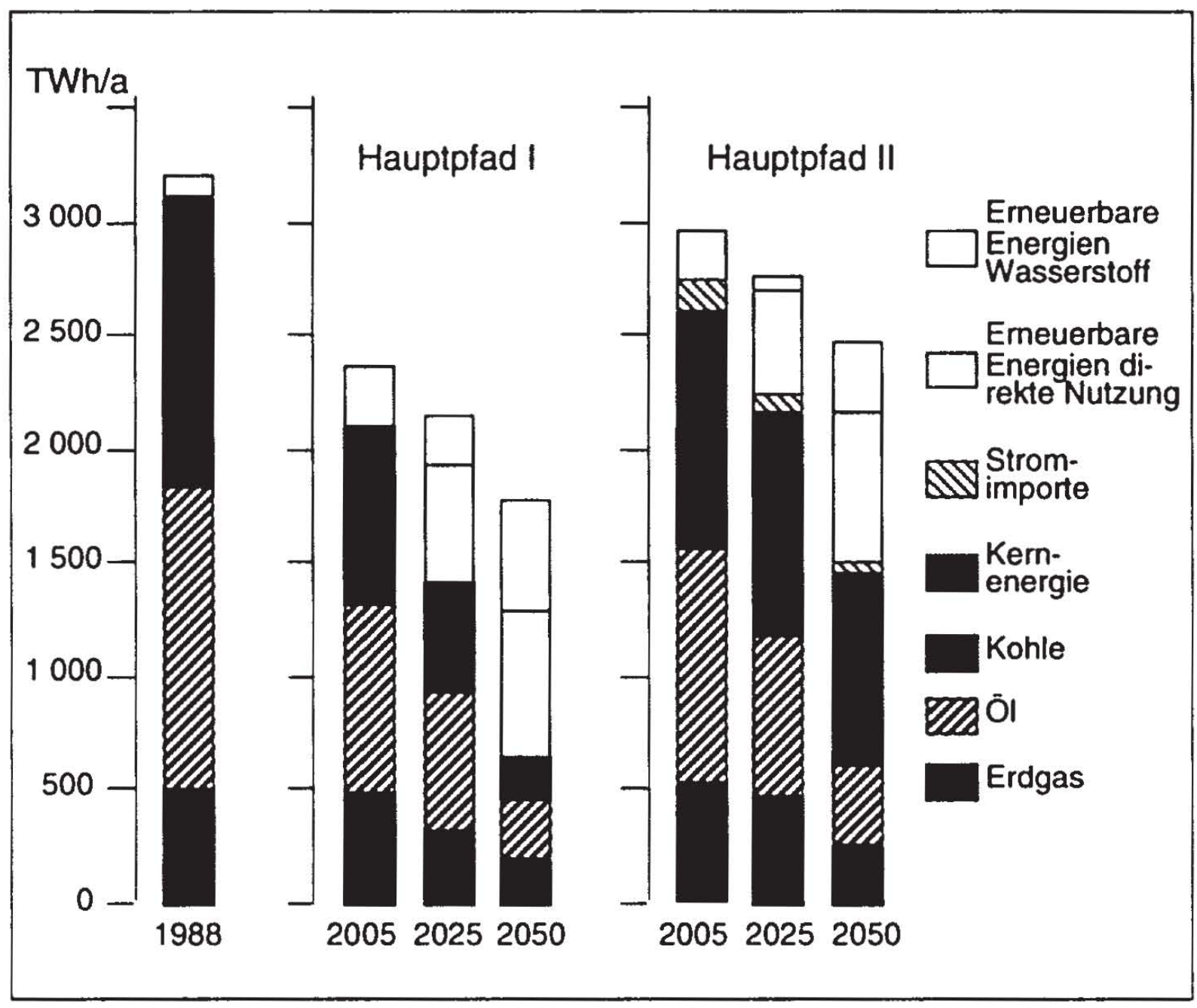

Bild 3 Primarenergieverbrauch und Energıetrager in den Szenarien der TA-Studie Solare Wasserstoffwirtschaft /5/

Die Analyse der Pfade ergab u. a. die nachfolgend beschriebenen Ergebnisse. Wesentliche Bedingungen für einen Einstieg in eine solare Wasserstoffwirtschaft sind:

J Notwendigkeit einer erheblichen $\mathrm{CO}_{2}$-Minderung,

J Verdoppelung der realen Energieträgerpreise bis 2005, z. B. durch Einführung einer $\mathrm{CO}_{2}$-Steuer von $200 \mathrm{DM} / \mathrm{tCO}_{2}$.

J 5 - 8-fach höhere reale Preise der fossilen Energieträger bis 2025,

$J$ erhebliche Kostensenkungen der regenerativen Energiesysteme (z. B. bei Photovoltaik um ca. $90 \%$ ),

J Abbau von Hemmnissen bei der Durchsetzung von Techniken zur rationellen Energieanwendung und zur Nutzung erneuerbarer Energieträger, 
- Weiterentwicklung der Schlüsseltechnologien für die solare Wasserstoffwirtschaft im Rahmen einer allgemein verstärkten Forschungsförderung für erneuerbare Energiequellen,

- keine Erzeugung von Wasserstoff aus Kernenergie im Hauptpfad II.

Folgende wesentliche Folgen des Aufbaus einer solaren Wasserstoffwirtschaft wurden identifiziert: Der Flächenbedarf für die solaren Systeme ist erheblich, aber nicht unrealistisch hoch. Neben vorhandenen $2800 \mathrm{~km}^{2}$ Dachflächen werden im Inland je nach Variante zwischen $700 \mathrm{~km}^{2}$ und $3900 \mathrm{~km}^{2}(0,3$ - 1,6\% der Landflächen der alten Bundesländer) benötigt. Im Ausland kommen maximal $3900 \mathrm{~km}^{2}$ (ca. 0,04\% der Fläche der Sahara) hinzu.

Es müssen erhebliche Investitionen getätigt werden. Insgesamt müssen beim Hauptpfad I 3850 Mrd. DM bis 2050 (ca. 47 Mrd. DM/a), beim Hauptpfad II $2621 \mathrm{Mrd}$. DM (ca. $30 \mathrm{Mrd}$. DM/a) investiert werden. Die realen Stromerzeugungskosten steigen im Hauptpfad I um das 3,3 - 4-fache, im Hauptpfad II um das 2,2 - 2,5-fache bis 2050 an.

Treibstoffe und Brennstoffe werden erheblich (um den Faktor 7 - 8!) teurer als derzeit. Die Umstrukturierung der Energieversorgung führt zu einer erhöhten Nachfrage bei der Investitionsgüterindustrie, Mineralölund Automobilindustrie schrumpfen.

In der Struktur der Energiewirtschaft ergeben sich große Änderungen. So wird es durch staatliche Eingriffe zu wesentlich höheren Energiepreisen kommen. Der Zubau von verschiedenen kleineren Energieanlagen zur Nutzung erneuerbarer Energieträger führt zu einer stärkeren Einspeisung kleinerer Anlagen in das Netz. Hierfür müssen Regeln bezüglich Technik und Einspeisevergütung aufgestellt werden. Schließlich wird die Bedeutung leitungsgebundener Energieträger (Strom, Gas, Fernwärme) zunehmen. Eine stärkere staatliche Regulierung ist die Folge.

Der Import von Strom oder Wasserstoff aus dem Ausland, insbesondere aus Nordafrika, kann zu außenpolitischen Problemen führen. So wird die Abhangigkeit von den OPEC Staaten zum Teil durch die Abhängigkeit von anderen sonnenreichen Staaten ersetzt.

Schließlich sind auch im Inland gesellschaftliche Auswirkungen denkbar. Insbesondere stellt sich die Frage, ob die Bevölkerung drastisch höhere Energiepreise akzeptiert. Auch die Akzeptanz von Solaranlagen mit hohem Flächenverbrauch und von zusätzlichen Stromleitungen ist offen.

Insgesamt hat die Studie eine ganze Reihe von Erkenntnissen über Bedingungen und Folgen einer solaren Wasserstoffwirtschaft gebracht. Allerdings sind auch eine Reihe von Fragen noch offen geblieben. So wurden die Auswirkungen einer solaren Wasserstoffwirtschaft auf die Umwelt 
und die menschliche Gesundheit nur rudimentär behandelt. Das gilt auch für den wichtigen Fragenkomplex, welche Auswirkungen die hohen Kosten der Energieversorgungssysteme auf die Volkswirtschaft haben. Zwar wurden die Kosten und auch die Aufteilung der Kosten auf die Verbraucher abgeschätzt, die Rückkopplung dieser Kosten auf die ökonomischen Parameter, z. B. das Wirtschaftswachstum, wurde jedoch nicht untersucht. Schließlich läßt sich mit den Ergebnissen dieser Studie allein die Frage, ob man denn politische Schritte zum Aufbau einer solaren Wasserstoffwirtschaft unternehmen soll, ob der Aufbau einer solaren Wasserstoffwirtschaft also wünschenswert ist, nicht beantworten.

Nimmt man an, daß Konsens über das Ziel einer weitreichenden $\mathrm{CO}_{2}$ Minderung erreicht wird, so bleibt dennoch die Frage offen, ob es nicht andere Möglichkeiten als die solare Wasserstoffwirtschaft gibt, mit denen sich eine vergleichbare $\mathrm{CO}_{2}$-Reduzierung mit geringeren Nachteilen realisieren läßt. Diese Frage läßt sich nur durch vergleichbare Analyse aller in Frage kommenden Alternativen beantworten (solche Alternativen sind z. B. die Entsorgung bzw. Speicherung von $\mathrm{CO}_{2}$, eine verstärkte Energieeinsparung, Fusion und fortgeschrittene Kernreaktoren, verstärkte Nutzung von Biomasse aus Energieplantagen, Maßnahmen zur Eingrenzung der Folgen des Treibhauseffekts).

Durch Betrachtung einer Technik im Rahmen einer technikinduzierten Technikfolgenabschätzung läßt sich daher über die Wünschbarkeit der Technik noch nichts aussagen, weil es für die Bereitstellung von Energiedienstleistungen in der Regel immer Alternativen gibt, deren Vor- und Nachteile gegeneinander abgewogen werden müssen.

\subsection{Bedarfsorientierte Technikfolgenabschätzung: Perspektiven der Energieversorgung Baden- Württembergs}

Das Reaktorunglück von Tschernobyl im Jahr 1986 war für alle, die in der Energiepolitik und Energiewirtschaft Verantwortung tragen, Anlaß, die Vorstellungen über die zukünftige Ausgestaltung der Energieversorgung und die Rolle der Kernenergie kritisch und in aller Gründlichkeit zu überprüfen.

In Wahrnehmung ihrer energiepolitischen Verantwortung hat daher die Landesregierung von Baden-Württemberg ein Gutachten in Auftrag ge- 
geben, das die Perspektiven der Energieversorgung und insbesondere die Möglichkeiten der Umstrukturierung der Energieversorgung BadenWürttembergs unter besonderer Berücksichtigung der Stromversorgung analysieren sollte $/ 6 /$. Angesichts dessen, daB die Untersuchung der Rolle bzw. der Vor- und Nachteile der Kernenergie allein nicht ausreicht, um energiepolitische Entscheidungen zu treffen, weil erst eine Abwägung der Option Kernenergie mit allen anderen Optionen und deren Vor- und Nachteilen Bewertungen zuläßt, sollte die Untersuchung nicht auf die Rolle der Kernenergie beschränkt bleiben. Vielmehr war es das Ziel des Gutachtens, technisch mögliche Wege einer langfristig gesicherten Energieversorgung Baden-Württembergs umfassend zu analysieren und hinsichtlich ihrer ökologischen, ökonomischen und gesellschaftlichen Auswirkungen zu bewerten. Das Gutachten sollte, aufbauend auf dem gegenwärtigen Stand des Wissens, Entscheidungshilfen für eine sachgerechte, an den Belangen der Bürger sowie einer modernen Industriegesellschaft und der Entwicklungsnotwendigkeiten der Dritten Welt orientierten Energiepolitik bereitstellen, um heute diejenigen Entscheidungen zu treffen bzw. Entwicklungen einleiten zu können, die zur langfristigen Sicherung einer ausreichenden, preisgünstigen, umwelt- und sozialverträglichen Energieversorgung notwendig sind. Nicht zuletzt hatte das Gutachten aber auch das Ziel, für die Energiediskussion eine solide, möglichst breit akzeptierte Faktengrundlage für die notwendigen energiepolitischen Abwägungs- und Wertungsprozesse bereitzustellen.

Um der umfassenden Aufgabenstellung des Gutachtens und der Komplexität des Energieproblems in der zur Verfügung stehenden Zeit gerecht $\mathrm{zu}$ werden, wurden die Untersuchungen auf die folgenden drei übergeordneten Themenfelder konzentriert:

(1) Analyse der Eigenschaften, Potentiale und Kosten der verschiedenen Energieversorgungsoptionen und der Möglichkeiten zur Reduzierung des Energieeinsatzes.

(2) Analyse unterschiedlicher technisch möglicher Wege der langfristigen Energieversorgung Baden-Württembergs (Szenarioanalyse).

(3) Analyse der gesamtwirtschaftlichen, ökologischen und gesellschaftlichen Implikationen verschiedener Energiesysteme.

Im Zentrum des ersten Themenfeldes stand die sorgfältige Erhebung des Wissens über die Techniken zur Bereitstellung und Nutzung von Energie sowie zur Energieeinsparung, die heute in absehbarer Zukunft zur Energieversorgung Baden-Württembergs beitragen können. Hierbei wurden der derzeitige Entwicklungsstand und die Entwicklungsperspektiven, die Kosten und Kostenentwicklungen, die Umweltauswirkungen sowie die möglichen quantitativen Versorgungsbeiträge der Techniken und Sy- 
steme zur Nutzung fossiler, nuklearer oder erneuerbarer Energiequellen sowie zur Reduzierung des Energieeinsatzes systematisch untersucht.

Für das zweite Themenfeld, die systematische Analyse möglicher energetischer Zukunftsperspektiven sowie der energiepolitischen Gestaltungsund Handlungsmöglichkeiten wurde der Szenario-Ansatz benutzt. Unter einem Szenario ist dabei die Beschreibung einer zukünftigen Entwicklung auf der Basis vernünftiger, in sich konsistenter Annahmen zu verstehen. Mit Hilfe verschiedener Szenarien wurde versucht,

J die Auswirkungen unterschiedlicher, exogener Einflußfaktoren auf die Energieversorgung Baden-Württembergs aufzuzeigen, deren Entwicklung unsicher ist und die primär nicht von den Entscheidungen über ein zukünftiges Energiesystem in Baden-Württemberg mitbestimmt werden wie z. B. die Entwicklung des Wirtschaftswachstums oder der Preise auf den Weltenergiemärkten;

J unterschiedliche Vorstellungen über die zukünftige Energieversorgung quantitativ darzustellen, um sie damit rational diskutierbar zu machen;

J die Möglichkeiten und Wege zur Erreichung energiepolitischer Ziele zu identifizieren sowie die Grenzen energiepolitischen Handelns zu ermitteln.

Im Rahmen der Szenarioanalysen kommt der Energienachfrageseite und ihren Beeinflussungsmöglichkeiten der gleiche Rang wie der Analyse der Versorgungsmöglichkeiten zu. Die mit den Szenarien beschriebenen möglichen zukünftigen Entwicklungen von Energiebedarf und Energieversorgung dürfen dabei nicht als Prognosen, also Vorhersage der zukünftigen Entwicklung mißverstanden werden. Es geht nicht darum, vorherzusagen, wieviele Kraftwerke im Jahr 2020 installiert sein werden oder wieviel Mineralöl im Jahr 2000 noch verbraucht wird - Aussagen, die in Anbetracht der bestehenden Unsicherheit ohnehin nicht möglich sind sondern darum, Informationen und Erkenntnisse über mögliche $\mathrm{Zu}$ kunftsentwicklungen und ihre Zusammenhänge und Konsequenzen zu gewinnen, die notwendig und hilfreich bei der Festlegung der heute zu treffenden Entscheidungen sind. Die technisch-energetische, ökonomische und emissionsseitige Quantifizierung der Szenarien wurde mit einem System von Energiemodellen vorgenommen. Die Modelle bildeten die Zusammenhänge und strukturellen Änderungen des Energiesystems von Baden-Württemberg energieträgerspezifisch ab und zwar ausgehend von den Determinanten des Energiebedarfs über den Energieeinsatz bei den Endverbrauchern in den Bereichen Industrie, Verkehr, Haushalte und Kleinverbraucher sowie die Umwandlung in Kraftwerken, Heizwerken, Raffinerien usw. bis hin zum Primärenergieeinsatz. 
Im Themenfeld drei wurden die Auswirkungen analysiert. Bei den gesamt- und einzelwirtschaftlichen Konsequenzen verschiedener Entwicklungen des Energiesystems in Baden-Württemberg waren die durch Preisveränderungen induzierten Anpassungsprozesse und Wirkungen auf das Wachstumspotential der Volkswirtschaft ebenso zu berücksichtigen wie die unterschiedlichen sektoralen Wettbewerbs- und Beschäftigungseffekte. Bei den ökologischen Auswirkungen bildeten die Entsorgungsaspekte sowie die Luftschadstoffemissionen und ihre Wirkungen auf die Natur und das Klima den Schwerpunkt. Darüber hinaus wurden Risiken für die menschliche Gesundheit, die mit der Nutzung verschiedener Stromerzeugungssysteme verbunden sind, vergleichend untersucht und überprüft, ob eine objektivierbare Beurteilung der Sozialverträglichkeit von Energiesystemen möglich ist und inwieweit dieses Kriterium operational für die Beurteilung der gesellschaftlichen Aspekte von Energiesystemen eingesetzt werden kann.

Um der fachlichen Breite der Aufgabenstellung des Gutachtens gerecht zu werden, waren mehr als achtzig Wissenschaftler und Experten aus achtzehn Institutionen direkt an den Arbeiten beteiligt. Darüber hinaus sind zwei Workshops durchgeführt worden, um Ergebnisse und Teilergebnisse der Arbeiten mit nicht an der Erarbeitung des Gutachtens beteiligten Fachleuten mit dem Ziel zu diskutieren, die wissenschaftliche Basis der Ergebnisse weiter abzusichern. Darüber hinaus haben zahlreiche Fachleute aus der Industrie, aus Verbänden, Behörden und wissenschaftlichen Institutionen mit Anregungen, Daten und kritischen Kommentaren zu den Gesamtergebnissen des Gutachtens beigetragen. Im folgenden sollen aus der Fülle der vorliegenden Ergebnisse lediglich einige wenige exemplarisch vorgestellt werden, um die Möglichkeiten in bezug auf die Technikfolgenabschätzung anzudeuten.

Um die Auswirkungen der verschiedenen, in der Studie zu untersuchenden energetischen Zukunftsperspektiven (Szenarien) beurteilen und vergleichen zu können, ist es hilfreich, sich als Vergleichsmaßstab eine Referenzentwicklung vorzugeben und daran die quantitativen Wirkungen und Veränderungen in den verschiedenen Szenarien aufzuzeigen. Die diesem Zweck dienenden Referenzszenarien sind also nichts weiter als eine Meßlatte für die anderen Szenarien. Sie sind nicht dadurch ausgezeichnet, daß sie im Sinne einer Prognose die wahrscheinlichsten Entwicklungen repräsentieren. Die Referenzszenarien, die im Rahmen des Gutachtens verwendet werden, sind dadurch charakterisiert, daß sie von einer Fortsetzung der bisherigen Energiepolitik und einem Fortbestehen der energiewirtschaftlichen Rahmenbedingungen ausgehen. Wegen der Unsicherheiten, die mit der zukünftigen Entwicklung exogener Bestimmungsfaktoren für Energiebedarf und Energieversorgung verbunden sind, reicht ein Re- 
ferenzszenario als Status quo-Variante nicht aus. Es wurden vielmehr drei Referenzszenarien erstellt, die sich insbesondere durch unterschiedliche Annahmen bezüglich des Wirtschaftswachstums und der Weltmarktpreise für Energieträger unterscheiden. Diese drei Szenarien sollen eine Bandbreite möglicher Entwicklungen, bei denen wesentliche Rahmenbedingungen, insbesondere energiepolitische Einwirkungen, konstant bleiben, aufzeigen.

Die drei Varianten des Referenzszenarios sind gekennzeichnet durch ein unterschiedliches Wachstum des Bruttoinlandproduktes $(0,6 \% / \mathrm{a}, 2 \%$ /a und 2,6\%/a). In drei Preisvarianten wurde ein Anstieg des Preises von Rohöl auf 26, 30 bzw. 48 Dollar pro Barrel angenommen. Als zusammenfassende Ergebnisse der sehr detaillierten Analyse der Entwicklung von Energieverbrauch und -versorgung in den Referenzszenarien zeigen Bild 4 und Bild 5 die Entwicklung des Endenergieverbrauchs und des Stromverbrauchs bis 2020 in Baden-Württemberg.

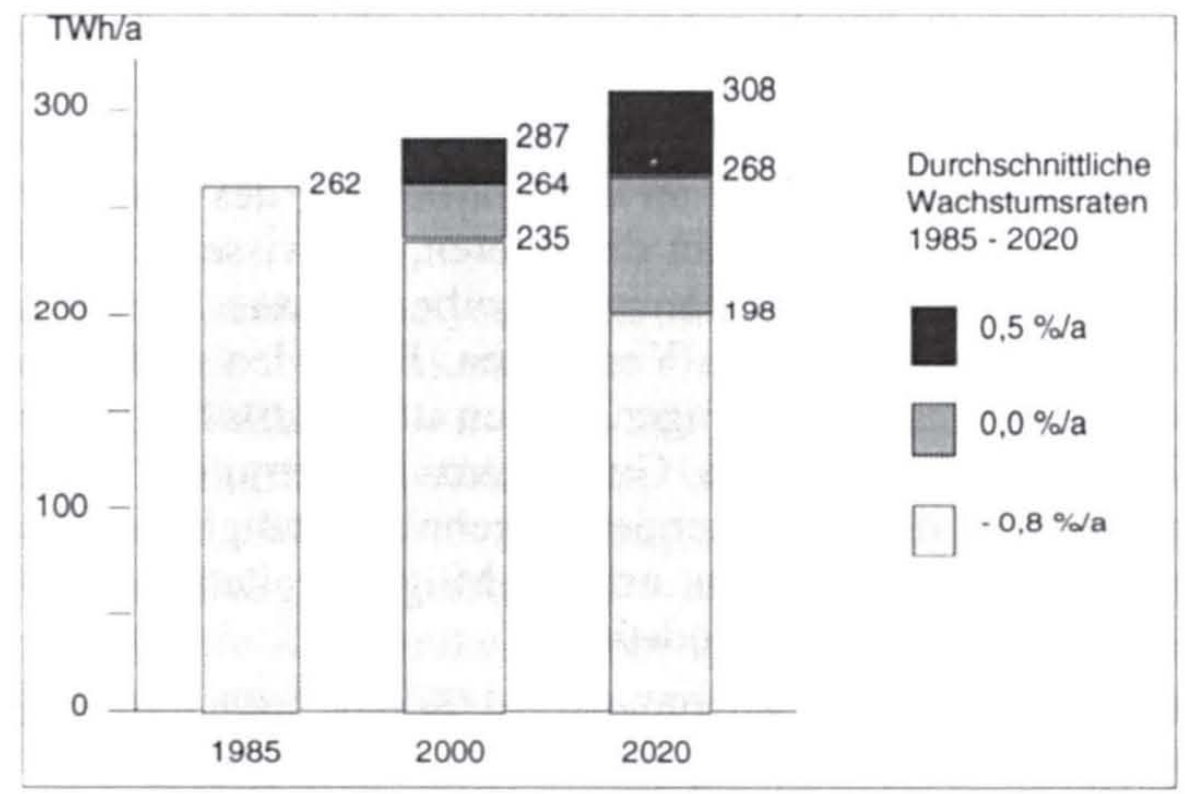

Bild 4 Entwicklung des Endenergieverbrauchs in Baden-Württemberg in den Referenzszenarien des Energiegutachten Baden-Württemberg /6/

Es zeigt sich, daß der Endenergieverbrauch unter Status-quo-Bedingungen eher stagnieren wird. Wachstumsraten wie vor 1973 werden nicht mehr erreicht. Die Wachstumsraten des Stromverbrauchs liegen deutlich höher als die des Endenergieverbrauchs. 


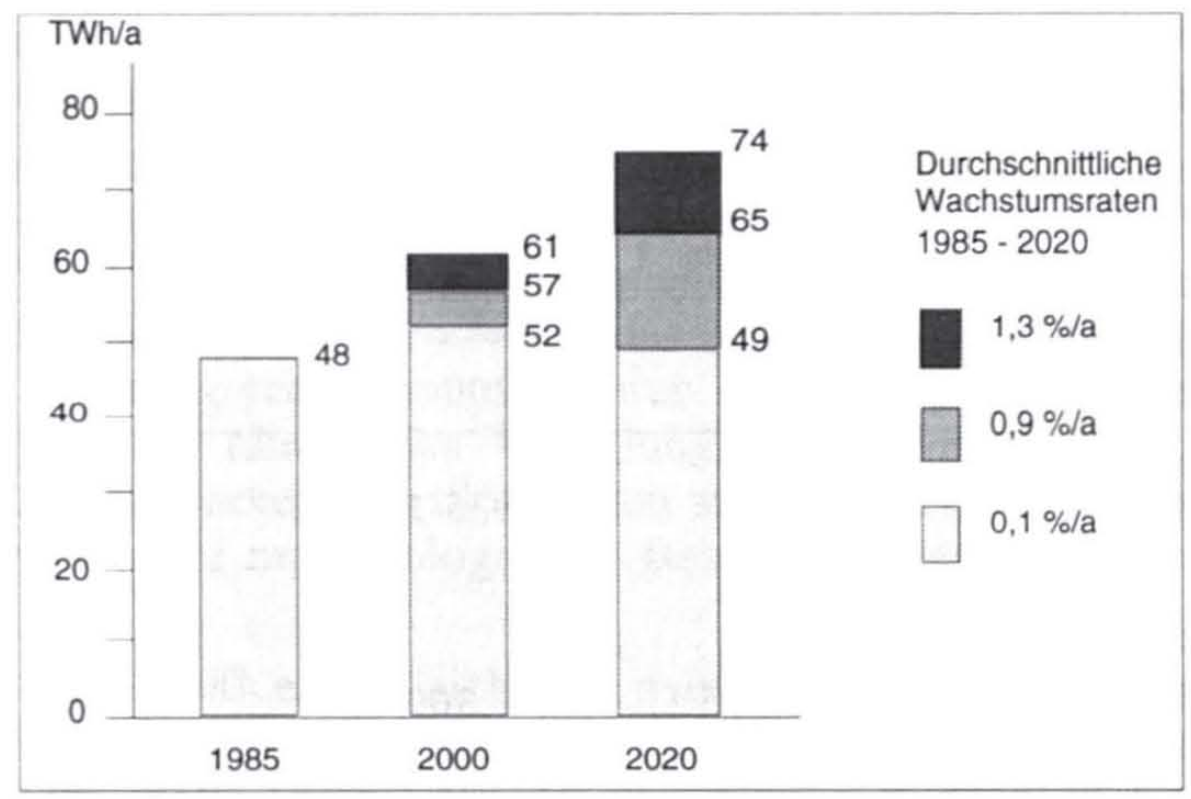

Bild 5 Entwicklung des Stromverbrauchs in Baden-Württemberg in den Referenzszenarien des Energiegutachten Baden-Württemberg /6/

Die Erstellung der Szenarien alternativer Entwicklungen des Energiesystems soll anhand des Szenarios verstärkter Ausbau erneuerbarer Energieträger erläutert werden. Betrachtet wurden die erneuerbaren Energiequellen und Nutzungssysteme, die nach dem gegenwärtigen Stand des Wissens für eine Energieerzeugung in Baden-Württemberg von Bedeutung sein können. Es sind dies

- die Wasserkraftnutzung,

๖ die Windenergienutzung,

- die photovoltaische Nutzung der Solarstrahlung,

- die thermische Nutzung der Solarstrahlung,

๑ die energetische Nutzung von Biomasse, Abfällen und Müll und

๖ die Nutzung geothermischer Energie.

Für jede dieser Energiequellen wurden das Energieangebot und die grundsätzlichen Erschließungsmöglichkeiten, die Techniken zur Nutzung des Energieangebots, die Energiebereitstellungskosten ausgewählter Nutzungssysteme und ihre zukünftige Entwicklung, die technischen und wirtschaftlichen Nutzungspotentiale und ihre Ausschöpfungsmöglichkeiten sowie die Umweltauswirkungen untersucht.

Typische Ergebnisse sollen am Beispiel der Windenergie erläutert werden. Tab. 2 zeigt die derzeitigen sowie die zukünftig erwarteten Kosten von drei Referenztechniken von Windenergiekonvertern (WEK) mit jeweils 
$75 \mathrm{~kW}, 200 \mathrm{~kW}$ und $1200 \mathrm{~kW}$. Die Stromerzeugungskosten sind insbesondere stark von der mittleren Windgeschwindigkeit abhängig.

\begin{tabular}{|c|c|c|c|}
\hline & Technik 1 & Technik 2 & Technik 3 \\
\hline Rotordurchmesser $(\mathrm{m})$ & 15.5 & 25 & 60 \\
\hline Nennleistung ( $k W$ ) & 75 & 200 & 1200 \\
\hline $\begin{array}{l}\text { Investitionskosten } \\
\text { (1 } 000 \text { DM) }\end{array}$ & $156 / 117$ & $635 / 385$ & $8400 / 4200$ \\
\hline $\begin{array}{l}\text { Spezifische Kosten: } \\
\text { - DM/m Rotorfläche } \\
\text { - DM/kW Nennleistung }\end{array}$ & $\begin{array}{l}821 / 615 \\
2080 / 1560\end{array}$ & $\begin{array}{ll}1 & 300 / 790 \\
3 & 175 / 1925\end{array}$ & $\begin{array}{lll}2 & 970 / 1 & 485 \\
7 & 000 / 3 & 500\end{array}$ \\
\hline $\begin{array}{l}\text { Stromerzeugungskosten } \\
\text { (DM/kWh) }\end{array}$ & & & \\
\hline bei $v=6,0 \mathrm{~m} / \mathrm{s}$ & $0.18 / 0.12$ & $0,24 / 0,13$ & $0.50 / 0,16$ \\
\hline $\mathrm{v}=4.5 \mathrm{~m} / \mathrm{s}$ & $0.29 / 0.20$ & $0,39 / 0,22$ & $0.92 / 0.30$ \\
\hline$v=3.5 \mathrm{~m} / \mathrm{s}$ & $0,48 / 0,32$ & $0,68 / 0.39$ & $2,09 / 0,68$ \\
\hline
\end{tabular}

Tab. 2 Kosten von Windenergiekonvertern (Referenztechniken) (linke Zahl: Kosten 1986 / rechte Zahl: Kosten 2000/2020)

Das Potential der Windenergienutzung hängt von den zur Verfügung stehenden Flächen und der Verteilung der Windgeschwindigkeit auf diesen Flächen ab. Maximal ein Viertel der Gebietsfläche Baden-Württembergs kommt überhaupt für eine Windenergienutzung in Frage (mittlere Windgeschwindigkeit $>3 \mathrm{~m} / \mathrm{s}$ ). Die gut geeigneten Gebiete (Jahresmittel der Windgeschwindigkeit $>4 \mathrm{~m} / \mathrm{s}$ ) betragen jedoch nur $6 \%$ der Gebietsfläche. Unter Beachtung ökologischer Gesichtspunkte und anderer Nutzungen stehen jedoch nur 10 bis $20 \%$ dieser Flächen für eine Windenergienutzung zur Verfügung. Die somit erzielbare maximale Elektrizitätserzeugung beträgt rd. $2400 \mathrm{GWh} / \mathrm{a}$, dazu wären rd. 16500 Windenergiekonverter mit einer Leistung von $200 \mathrm{~kW} \mathrm{zu}$ installieren.

Durch Verknüpfung der technischen Daten und Kosten mit der Verteilung der Windgeschwindigkeit läßt sich das Potential in Abhängigkeit von den Stromerzeugungskosten ermitteln - als Ergebnis erhält man die in Bild 6 gezeigte Potential-Kosten-Funktion. Eine solche Funktion erlaubt die Ermittlung des wirtschaftlichen Beitrags von Windenergie in Abhängigkeit von den anlegbaren Vergleichskosten von Alternativen zur 
Stromerzeugung. Die erforderliche Höhe und die Wirkungen von Fördermaßnahmen lassen sich ebenfalls anhand dieser Kurve ableiten.

Nach Durchführung ähnlicher Analysen für die anderen erneuerbaren Energieträger lassen sich Aussagen zum technischen und wirtschaftlichen Potential aller erneuerbaren Energiequellen machen. Das technische Potential ist dabei dasjenige Potential, das unter Berücksichtigung der verfügbaren Nutzungstechniken und ihrer Nutzungsgrade, des zeitlichen Verlaufs und der räumlichen Verteilung von Energieangebot und -bedarf, der Verfügbarkeit von Standorten und konkurrierenden Nutzungen sowie struktureller und ökologischer Beschränkungen technisch nutzbar ist.

Für das Jahr 2000 ergibt sich ein maximaler Versorgungsbeitrag zu $6300 \mathrm{GWh} / \mathrm{a}$ Strom und ca. $10500 \mathrm{GWh} / \mathrm{a}$ an sonstiger Endenergie und für das Jahr 2020 lauten die entsprechenden Zahlen $12500 \mathrm{GWh} / \mathrm{a}$ Elektrizität und $45100 \mathrm{GWh} / \mathrm{a}$ sonstige Endenergie. Dies entspräche einem Anteil am Endenergieverbrauch beim mittleren Referenzszenario von $6,4 \%$ im Jahr 2000 und $22 \%$ im Jahr 2020.

Im einzelnen ergeben sich folgende Beiträge erneuerbarer Energieträger am technischen Potential im Jahr 2020:

$\begin{array}{llr}\square & \text { Solare Strahlung } & 28,5 \mathrm{TWh} ; \\ \square & \text { Windenergie } & 2,4 \mathrm{TWh} ; \\ \square & \text { Wasserkraft } & 6,3 \mathrm{TWh} ; \\ \square & \text { Biomasse und Müll } & 16,3 \mathrm{TWh} ; \\ \square & \text { Geothermie } & 4,5 \mathrm{TWh} .\end{array}$

Das wirtschaftliche Potential beschreibt den Beitrag von Energieversorgungssystemen bzw. Einsparmaßnahmen, der im Vergleich zu konkurrierenden Systemen zu gleichen oder geringeren Kosten bereitgestellt werden kann. Es hängt naturgemäß von der Preisentwicklung der fossilen Energieträger $a b$ und beträgt je nach angenommener Preisentwicklung zwischen 15 und 50 TWh/a (5,7 - $15 \%$ des Endenergieverbrauchs). Davon werden ca. 7,4 TWh bereits heute genutzt. 


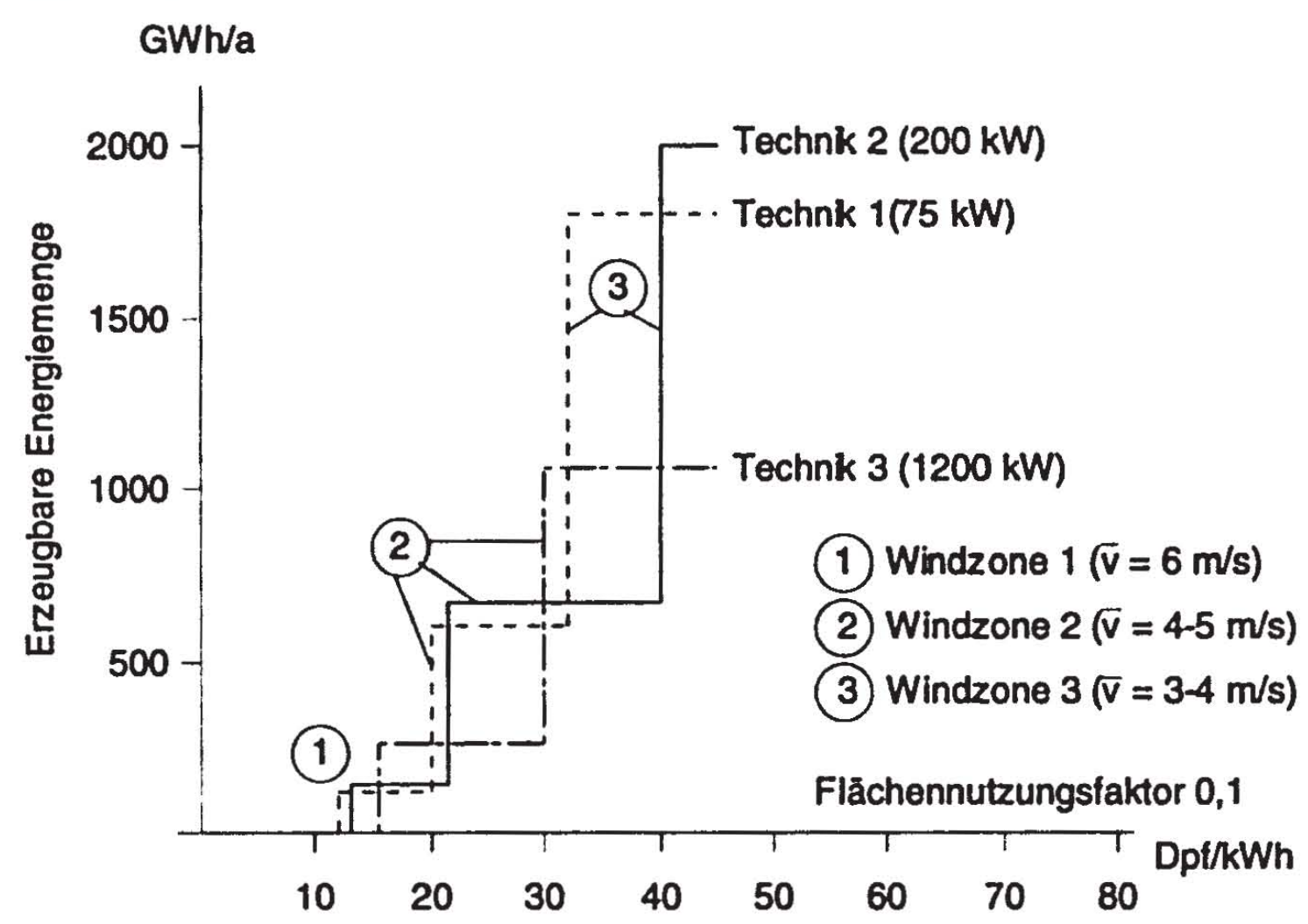

gemittelte reale Stromgestehungskosten über Nutzungsdauer

Bild 6 Windenergiepotential in Abhängigkeit von den Stromgestehungskosten

Um die Auswirkungen (Folgen) einer verstärkten Nutzung erneuerbarer Energieträger abzuschätzen, wurde ein Szenario max. Ausschöpfung erneuerbarer Energieträger erstellt, das insbesondere den Einsatz des gesamten ermittelten technischen Potentials der emeuerbaren Energieträger vorsieht. Die Analyse dieses Szenarios zeigt u. a. folgende Vor- und Nachteile: Es ergeben sich jährliche Minderemissionen in folgender Höhe (in Klammern ist der Anteil der Minderung an den gesamten Emissionen angegeben):

\begin{tabular}{|c|c|c|c|}
\hline & $\mathrm{SO}_{2}$ & $\mathrm{NO}_{\mathrm{x}}$ & $\mathrm{CO}_{2}$ \\
\hline 2000 & $-2,4 \mathrm{kt}(2 \%)$ & $-2,1 \mathrm{kt}(1 \%)$ & $-2,8 \mathrm{Mio} . \mathrm{t}(4 \%)$ \\
\hline 2020 & $-12,2 \mathrm{kt}(13 \%)$ & $-9,3 \mathrm{kt}(3 \%)$ & $-11,4 \mathrm{Mio} . \mathrm{t}(17 \%)$ \\
\hline
\end{tabular}


Diesen Minderungsemissionen stehen jedoch Mehrkosten gegenüber. So betragen allein die Mehrkosten der Stromerzeugung etwa 500 Mio. DM/a bzw. $1 \mathrm{Pf} / \mathrm{kWh}_{\mathrm{el}}$.

Ähnliche Analysen wie für erneuerbare Energieträger wurden auch für die Möglichkeiten

a zur rationellen Energieverwendung und Energieeinsparung,

a zur Kraft-Wärme-Kopplung (zentral und dezentral),

a der Kernenergie und

】 von Energieversorgungsoptionen für das nächste Jahrhundert (Kernfusion, Import von Elektrizität und Wasserstoff aus solarer Energietechnik, Methanol und Wasserstoff als Sekundärenergieträger)

durchgeführt. Diskutiert wurden auch Aspekte der Sozialverträglichkeit; allerdings konnten hier wegen der vorhandenen Probleme, Kriterien für die Bestimmung des Ausmasses der Sozialverträglichkeit im Einzelfall zu bestimmen, keine quantitativen Aussagen gemacht werden. Mit den Ergebnissen des Energiegutachtens wurde eine breite Faktengrundlage zur Bewertung von Energietechniken und eine Grundlage für Entscheidungen zur Sicherung einer ausreichenden, umwelt- und sozialverträglich und kostengünstigen Energieversorgung vorgelegt. Die Ergebnisse wurden in zahlreichen Diskussionsveranstaltungen und Seminaren mit Vertretern der baden-württembergischen Ministerien, verschiedener gesellschaftlicher Gruppen, der Energieversorgungsunternehmen und in der Öffentlichkeit diskutiert. Sie wurden bei einer ganzen Reihe von energiepolitischen Entscheidungen und Initiativen der Landesregierung berücksichtigt.

\subsection{Folgeninduzierte TA-Studie: Möglichkeiten zur Minderung von $\mathrm{CO}_{2}$ in der Bundesrepublik Deutschland}

Anlaß, über eine Umstrukturierung der Energieversorgung nachzudenken, besteht vor allem dann, wenn

a Techniken zur Energieumwandlung oder zur rationellen Energieverwendung neu oder weiter entwickelt wurden und deren Rolle im Energiesystem zu definieren ist oder wenn

- erhebliche Folgen der derzeitigen Energieversorgung deutlich werden, z. B. auf Grund von Beobachtungen von Schäden wie den neu- 
artigen Waldschäden oder von neuen wissenschaftlichen Erkenntnissen wie etwa über die Folgen des Anstiegs der Konzentration der Treibhausgase in der Atmosphäre.

Im letzteren Fall ist es erforderlich, tragfähige Lösungen bzw. Lösungsansätze für die neu erkannten Probleme zu erarbeiten. Dazu sind im Rahmen einer folgeninduzierten Technikfolgenabschätzung alle aus heutiger Kenntnis denkbaren Handlungsmöglichkeiten zu analysieren, d. h. es ist der Beitrag dieser Möglichkeiten zur Bewältigung der Probleme im Zeitablauf zu quantifizieren sowie deren unerwünschte Nebeneffekte, Risiken und Kosten abzuschätzen.

Dies soll am Beispiel des bereits erwähnten Problems der Klimaänderungen durch die Emissionen von Treibhausgasen verdeutlicht werden. Dabei soll auf den Kenntnisstand der Klimaforschung und auf die zu erwartenden Klimaänderungen hier nicht eingegangen werden. Trotz der hier noch bestehenden Wissenslücken wird im weiteren davon ausgegangen, daB der gegenwärtige Wissensstand und die Indizien für eine anthropogene Klimaveränderung so weitreichend sind, da $B$ zumindest aus Vorsorgegründen Gegenmaßnahmen eingeleitet werden müssen, um die drohenden Klimaveränderungen in tolerierbaren Grenzen zu halten.

Um die Klimaänderungen und ihre Konsequenzen auf ein tolerierbares $\mathrm{Maß}$ zu begrenzen, hat die Weltkonferenz The Changing Atmosphere von Toronto gefordert, die weltweiten $\mathrm{CO}_{2}$-Emissionen bis zum Jahr 2005 um $20 \%$ und bis zum Jahr 2050 um $50 \%$ gegenüber dem Niveau des Jahres $1987 \mathrm{zu}$ reduzieren und die zweite Weltklimakonferenz von Genf weist daraufhin, daß es notwendig wäre die weltweiten $\mathrm{CO}_{2}$-Emissionen kontinuierlich um $1 \%$ pro Jahr zu reduzieren, um bis zur Mitte des nächsten Jahrhunderts den Anstieg der atmosphärischen $\mathrm{CO}_{2}$-Konzentration auf ein Niveau zu begrenzen, das $50 \%$ über dem der vorindustriellen Zeit liegt. In ähnlicher Weise sind auch die Freisetzungen der anderen Treibhausgase zu vermindern. Diese Reduktionen der globalen $\mathrm{CO}_{2}$-Emissionen bzw. die damit verbundenen Einschränkungen des Verbrauchs fossiler Energieträger sind dabei vor dem Hintergrund zu sehen, daß nahezu alle Energieprognosen von einem weiteren Anstieg des weltweiten Verbrauchs an fossilen Energieträgern ausgehen.

Was aber bedeuten diese globalen Minderungsziele für die einzelnen Staaten? Welche Treibhausgasemissionsminderungen resultieren daraus für die Bundesrepublik, damit sie ihren Beitrag zur Erreichung der globalen Ziele leistet?

Einen allgemeinen akzeptierten Schlüssel zur Ableitung nationaler Treibhausgasminderungsziele gibt es bisher nicht. Angesichts des Faktums, da $\beta$ die energiebedingte Freisetzung von Treibhausgasen in der 
Vergangenheit nahezu ausschließlich durch die Industrieländer erfolgt ist, die auch heute noch für rund $75 \%$ der $\mathrm{CO}_{2}$-Emissionen verantwortlich sind, werden sie den Hauptbeitrag zur Minderung der Treibhausgasemissionen leisten müssen. Eine erste Orientierung über die Größenordnung der $\mathrm{CO}_{2}$-Reduktion in unserem Land zur Erreichung der zuvor genannten globalen Minderungsziele mag die folgende einfache Überlegung geben. Um die Zielvorgaben der Toronto-Konferenz aus dem Jahr $1988 \mathrm{zu}$ erreichen, wären die weltweiten $\mathrm{CO}_{2}$-Emissionen des Jahres 1987 in Höhe von rd. $20 \mathrm{Mrd}$. $\mathrm{zu}$ verringern. Bei rd. 6,5 Mrd. Menschen im Jahr 2005 und rd. 10 Mrd. Menschen im Jahr 2050 würden diese Minderungsziele bedeuten, daß im Weltdurchschnitt jeder Erdenbürger dann 2,5 bzw. 1 t $\mathrm{CO}_{2}$ pro Jahr durch die Nutzung fossiler Energieträger freisetzen dürfte.

In der Bundesrepublik Deutschland betrugen die $\mathrm{CO}_{2}$-Emissionen je Einwohner im Jahr $1987 \mathrm{rd} .12 \mathrm{t}$ und in der ehemaligen DDR rd. $21 \mathrm{t}$. Gleiches Emissionsrecht vorausgesetzt, müßten wir also unsere $\mathrm{CO}_{2}$ Emissionen bis 2005 um weit mehr als $50 \%$ und bis zur Mitte des nächsten Jahrhunderts um mehr als $90 \%$ reduzieren. Diese Zahlen mögen zum einen die Dimension der notwendigen Umstrukturierung unserer vornehmlich auf fossilen Energieträgern beruhenden Energieversorgung zur Erreichung eines klimaverträglichen Energiesystems umreißen und zum anderen andeuten, mit welchen Reduktionsforderungen an die Industrieländer, z. B. von Seiten der Entwicklungsländer, im Rahmen der internationalen Verhandlungen zur Erreichung einer Konvention über den Schutz der Erdatmosphäre zu rechnen ist.

Unter Berücksichtigung der berechtigten Belange der Entwicklungsländer ergibt sich, daß die von der Bundesregierung angestrebte Minderung der $\mathrm{CO}_{2}$-Emissionen um $25 \%$ bis zum Jahr 2005 bzw. die von der Enquête-Kommission Vorsorge zum Schutz der Erdatmosphäre geforderte Reduktion um $30 \%$ nicht ausreichen werden, um die weltweiten Zielvorgaben der Toronto-Konferenz zu erreichen.

Unabhängig von dem letztendlich notwendigen Umfang der Treibhausgasminderung kommt bei der Formulierung von energiepolitischen Strategien und Konzepten zur Erreichung einer klimaverträglichen Energieversorgung der Differenzierung zwischen dem technisch Möglichen, dem wirtschaftlich Darstellbaren und dem ökologisch Effizienten eine besondere Bedeutung zu. Rein technisch gesehen stehen uns zumindest auf längere Sicht sehr weitgehende Treibhausgasminderungsmöglichkeiten zur Verfügung. Aber nicht alles was technisch machbar ist, ist auch wirtschaftlich darstellbar und schon gar nicht effizient im Sinne der Nutzung 
knapper verfügbarer Ressourcen zur Vermeidung von Klimaveränderungen.

Eine Politik, die die Klimagefahren auf ein tolerierbares Maß eingrenzen will, ist auf ein gleichgerichtetes Handeln aller Staaten angewiesen. Dies wird wohl nur zu erreichen sein, wenn die Lasten gerecht verteilt und so gering wie möglich sind, damit insbesondere die Länder der Dritten Welt auch ihre anderen, ihnen derzeit viel wichtigeren Entwicklungsziele erreichen können. Aus diesem Grund gewinnen kosteneffiziente $\mathrm{CO}_{2}$-Reduktionsmaßnahmen ihre große Bedeutung. Anders ausgedrückt, eine klimaverträgliche Begrenzung der Treibhausgasemissionen wird wohl nur erreicht werden können, wenn die dafür verfügbaren, begrenzten Aufwendungen streng nach dem ökonomischen Prinzip verwendet werden, mit jeder aufgewendeten Mark eine möglichst hohe Treibhausgasminderung zu erreichen. Dies ist ein zentrales Kriterium für die Erarbeitung von Strategien und Konzepten zur Abwendung der Klimagefahren.

Im folgenden wird nun auf die Möglichkeiten zur Minderung der $\mathrm{CO}_{2}$ Emissionen in der Bundesrepublik Deutschland (ohne die Gebiete der ehemaligen DDR) näher eingegangen. Dabei werden Untersuchungsergebnisse verwendet, die im Rahmen eines Studienprogramms für die Enquête-Kommission Vorsorge zum Schutz der Erdatmosphäre des Deutschen Bundestages /1/ erarbeitet wurden. Als Bezugszeitpunkte für quantitative Aussagen dienen dabei die Jahre 1987 und 2005. Die nachfolgend erläuterten Ergebnisse sind jedoch nur als erste Stufe einer umfassenden Technikfolgenabschätzung zu verstehen, da wichtige Aspekte wie z. B. die Umweltauswirkungen der $\mathrm{CO}_{2}$-Minderungsmaßnahmen, die ökonomischen Auswirkungen der $\mathrm{CO}_{2}$-Minderungsstrategien und sonstige Aspekte wie Auswirkungen auf die Versorgungssicherheit oder die internationalen Energiemärkte nicht mit einbezogen wurden.

\section{$\mathrm{CO}_{2}$-Minderungsmöglichkeiten}

Im Jahr 1989 betrugen die energiebedingten $\mathrm{CO}_{2}$-Emissionen in der Bundesrepublik Deutschland (ohne die neuen Bundesländer) rd. 700 Mio. $\mathrm{CO}_{2}$. Davon entfielen auf den Umwandlungssektor und hier insbesondere die Stromerzeugung rd. 295 Mio. t oder $42 \%$. Die Sektoren Haushalte und Kleinverbraucher, Industrie und Verkehr waren mit $17 \%$ bis $20 \%$ an den Gesamtemissionen beteiligt.

Grundsätzlich lassen sich die energiebedingten $\mathrm{CO}_{2}$-Freisetzungen in die Atmosphäre reduzieren durch 
口 eine Minderung des Verbrauchs fossiler Energieträger durch rationellere Energieverwendung oder Energieeinsparung,

- den Ersatz fossiler Energieträger durch $\mathrm{CO}_{2}$-freie Energiequellen wie die Kernenergie und die erneuerbaren Energiequellen,

- eine Substitution kohlenstoffreicher (z. B. Kohle) durch kohlenstoffärmere (z. B. Erdgas) fossile Energieträger sowie

- durch eine Vermeidung der Freisetzung des bei der Verbrennung fossiler Energieträger entstehenden $\mathrm{CO}_{2}$ in die Atmosphäre $\left(\mathrm{CO}_{2}\right.$ Rückhaltung und Entsorgung).

\section{Rationelle Energienutzung und Energieeinsparung}

Auf allen Stufen der Prozeßkette von der Energiegewinnung über die Umwandlung bis zur Nutzung beim Verbraucher konnten in den letzten Jahren deutliche Fortschritte in bezug auf eine Steigerung der Energieeffizienz erzielt werden. Gleichwohl gilt die Feststellung, daß mit den in der Vergangenheit erreichten Nutzungsgradverbesserungen und Effizienzsteigerungen die technischen Möglichkeiten zur Minderung des Energieverbrauchs bei gleicher Energiedienstleistung, d. h. ohne Konsumverzicht, noch keineswegs ausgeschöpft sind.

Im Rahmen der Arbeiten für die Enquête-Kommission Vorsorge zum Schutz der Erdatmosphäre wurden die aus heutiger Sicht technisch möglichen Energieeinsparungen und die damit verbundenen $\mathrm{CO}_{2}-\mathrm{Minde}$ rungen abgeschätzt. Bild 7 zeigt diese technisch möglichen $\mathrm{CO}_{2}$-Reduktionspotentiale für verschiedene Verwendungsbereiche der Energie. In Summe belaufen sie sich auf 35 bis $45 \%$ der $\mathrm{CO}_{2}$-Emissionen des Jahres 1987.

Die Realisierung dieser durch Einsparmaßnahmen rein technisch möglichen $\mathrm{CO}_{2}$-Minderungen ist dabei je nach Maßnahme mit einem unterschiedlichen Aufwand verbunden, der in der Regel mit einer steigenden Ausschöpfung des technischen $\mathrm{CO}_{2}$-Minderungspotentials überproportional ansteigt, d. h. die Grenzkosten der $\mathrm{CO}_{2}$-Minderung nehmen zu. Eine Quantifizierung der $\mathrm{CO}_{2}$-Minderungskosten durch Energieeinsparung oder gar die Angabe von $\mathrm{CO}_{2}$-Minderungs-Kosten-Funktionen der verschiedenen Energieeinsparmaßnahmen ist gegenwärtig nur für Teilbereiche möglich. 


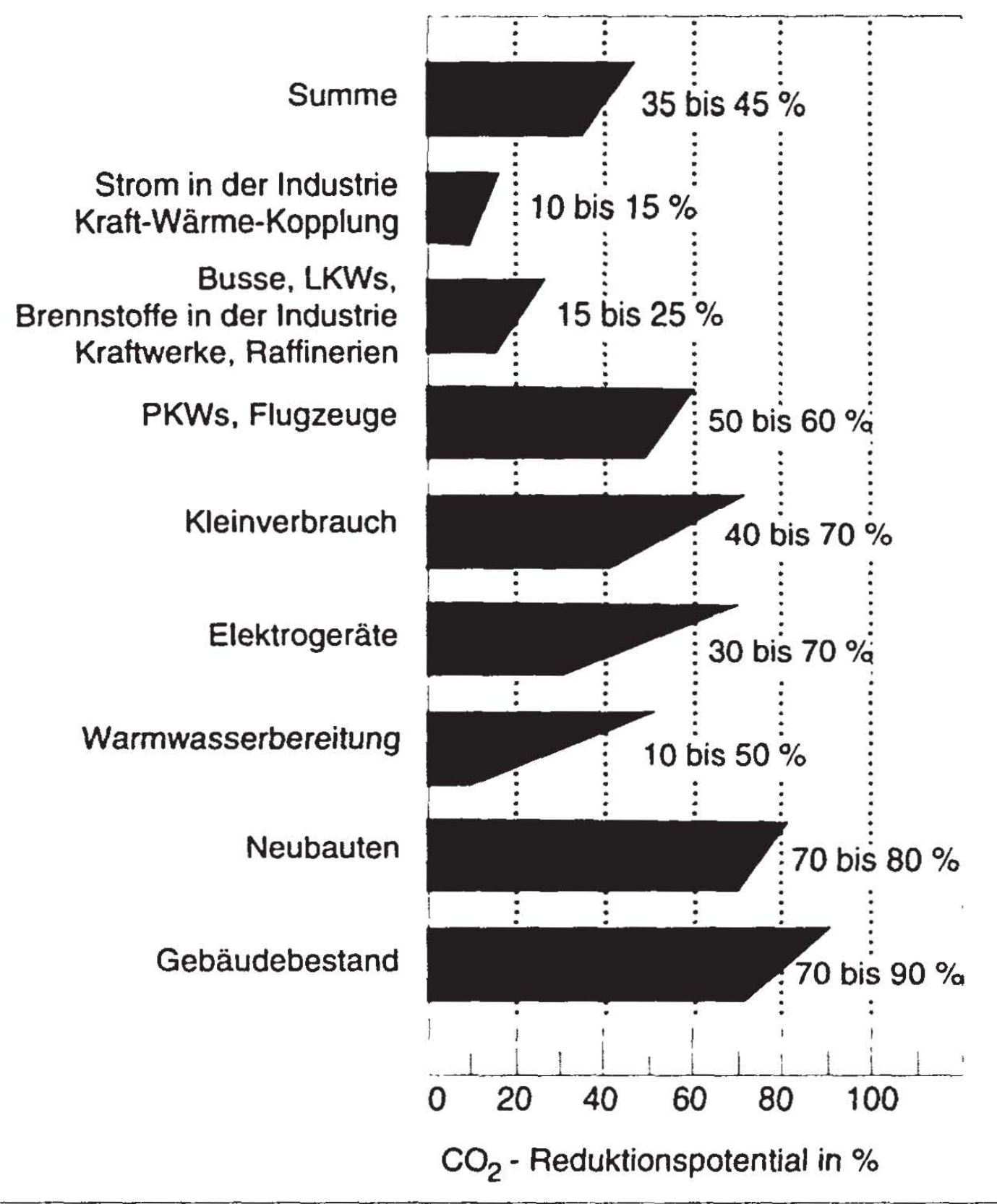

Bild 7 Technische Potentiale der Energieeinsparung in der BRD in Prozent des Energieverbrauchs im Jahre 1987

In Tab. 3 sind für einige ausgewählte Energieeinsparmaßnahmen die damit verbundenen $\mathrm{CO}_{2}$-Minderungskosten angegeben. Als $\mathrm{MaB}$ für die Effizienz der $\mathrm{CO}_{2}$-Minderung werden dabei die spezifischen $\mathrm{CO}_{2}$-Minderungskosten verwendet, die den Aufwand in DM angeben, um die Emissionen einer Tonne $\mathrm{CO}_{2}$ zu vermeiden. Die hier und im weiteren genannten spezifischen $\mathrm{CO}_{2}$-Minderungskosten sind dabei anhand einer für das Jahr 2005 unterstellten Energiepreissituation ermittelt worden, die da- 
durch gekennzeichnet ist, daß die Importpreise von Öl, Erdgas und Kohle gegenüber 1987 um rund $50 \%$ ansteigen, während die Strompreise nur leicht zunehmen. Die in Tab. 3 aufgeführten negativen Minderungskosten bedeuten, daß unter den getroffenen Preisannahmen für das Jahr 2005 diese Einsparmaßnahmen auch ohne eine Bewertung ihrer $\mathrm{CO}_{2}$ Minderung wirtschaftlich sind, d. h. die Kostenersparnis durch geringeren Energieverbrauch ist, über die Nutzungsdauer betrachtet, größer als der Aufwand für die Energieeinsparmaßnahme. Die große Bandbreite der angegebenen spezifischen $\mathrm{CO}_{2}$-Minderungskosten einer Maßnahme resultiert zum einen aus den unterschiedlichen Einsatzbedingungen ( $\mathrm{z}$. B. Leistungsgröße, jährliche Nutzungsstunden, usw.) und zum anderen aus der Art des eingesparten fossilen Energieträgers (Kohle, Mineralöl oder Gas). Dennoch machen die Zahlen eindrucksvoll deutlich, daß mit demselben Kostenaufwand je nach durchgeführter Energieeinsparmaßnahme viel oder wenig Minderung des $\mathrm{CO}_{2}$-Ausstoßes erreicht werden kann.

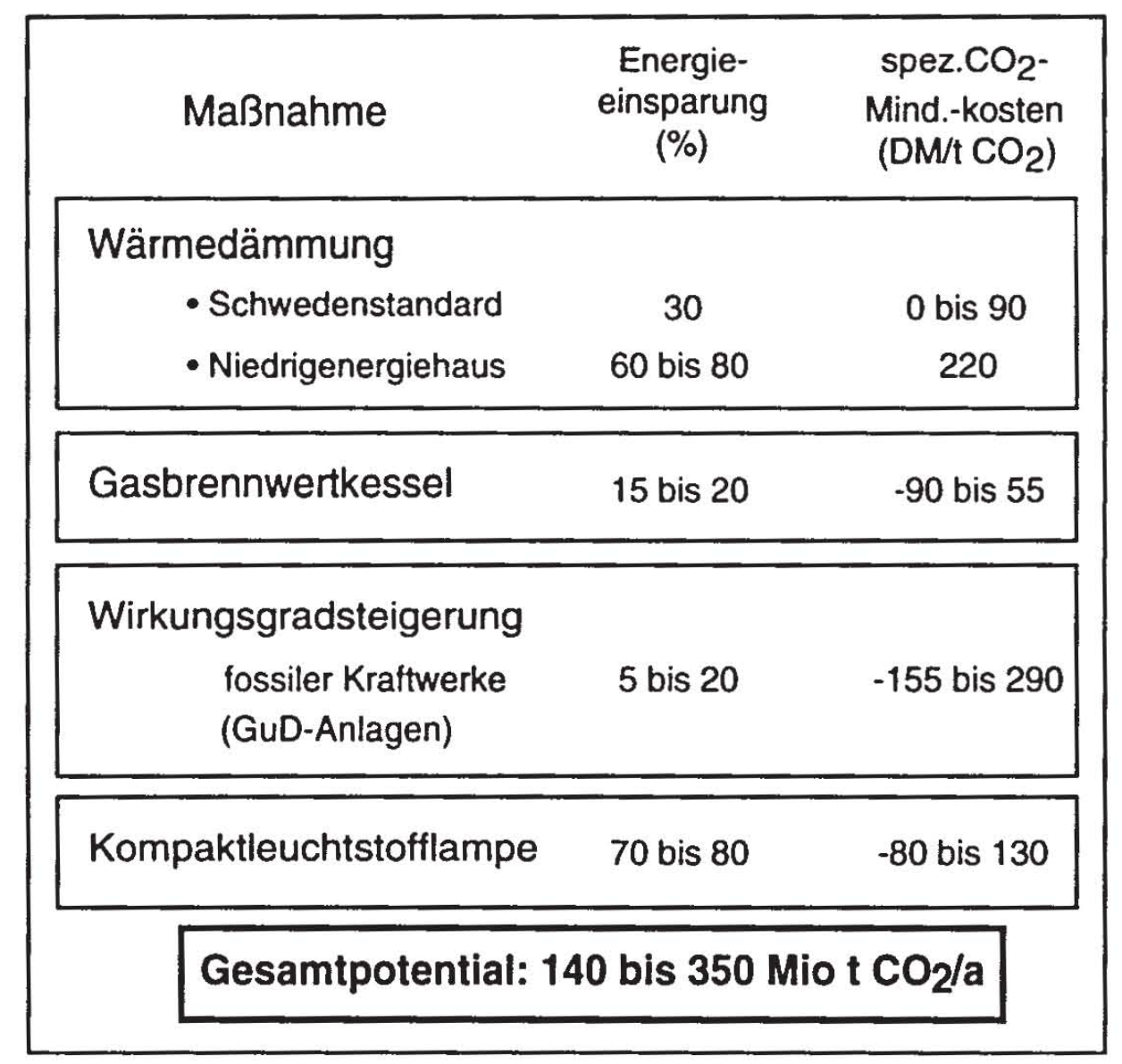

Tab. 3 Energieeinsparung und spezifiche $\mathrm{CO}_{2}$-Minderungskosten einiger Energiesparmaßnahmen 
In ähnlicher Form wie die Maßnahmen zur rationalen Energieanwendung werden auch die Maßnahmen:

a Austausch fossiler Energieträger untereinander,

- Kernenergie,

כ Erneuerbare Energieträger und

a Rückhalte- und Entsorgungsmöglichkeiten von $\mathrm{CO}_{2}$

untersucht. In Bild 8 sind die ermittelten technischen $\mathrm{CO}_{2}$ - Reduktionspotentiale der im Prinzip zur Verfügung stehenden $\mathrm{CO}_{2}$-Minderungsmöglichkeiten im Vergleich dargestellt. Die einzelnen Potentialangaben bezeichnen die $\mathrm{CO}_{2}$-Minderungen, die aus technischer Sicht unter Vernachlässigung ökonomischer und sonstiger Aspekte für die Bundesrepublik Deutschland (ohne die Gebiete der ehemaligen DDR) mittels erheblicher Anstrengungen gegebenenfalls bis zum Jahr 2005 erreichbar wären. Die technischen $\mathrm{CO}_{2}$-Minderungspotentiale der einzeinen Optionen können nicht zu einem Gesamtpotential aufsummiert werden, da sie sich teilweise auf denselben fossilen Energieverbrauch beziehen. Dennoch erscheint die Feststellung gerechtfertigt, daß für die Bundesrepublik Deutschland bereits mittelfristig nennenswerte $\mathrm{CO}_{2}$-Minderungen technisch möglich erscheinen.

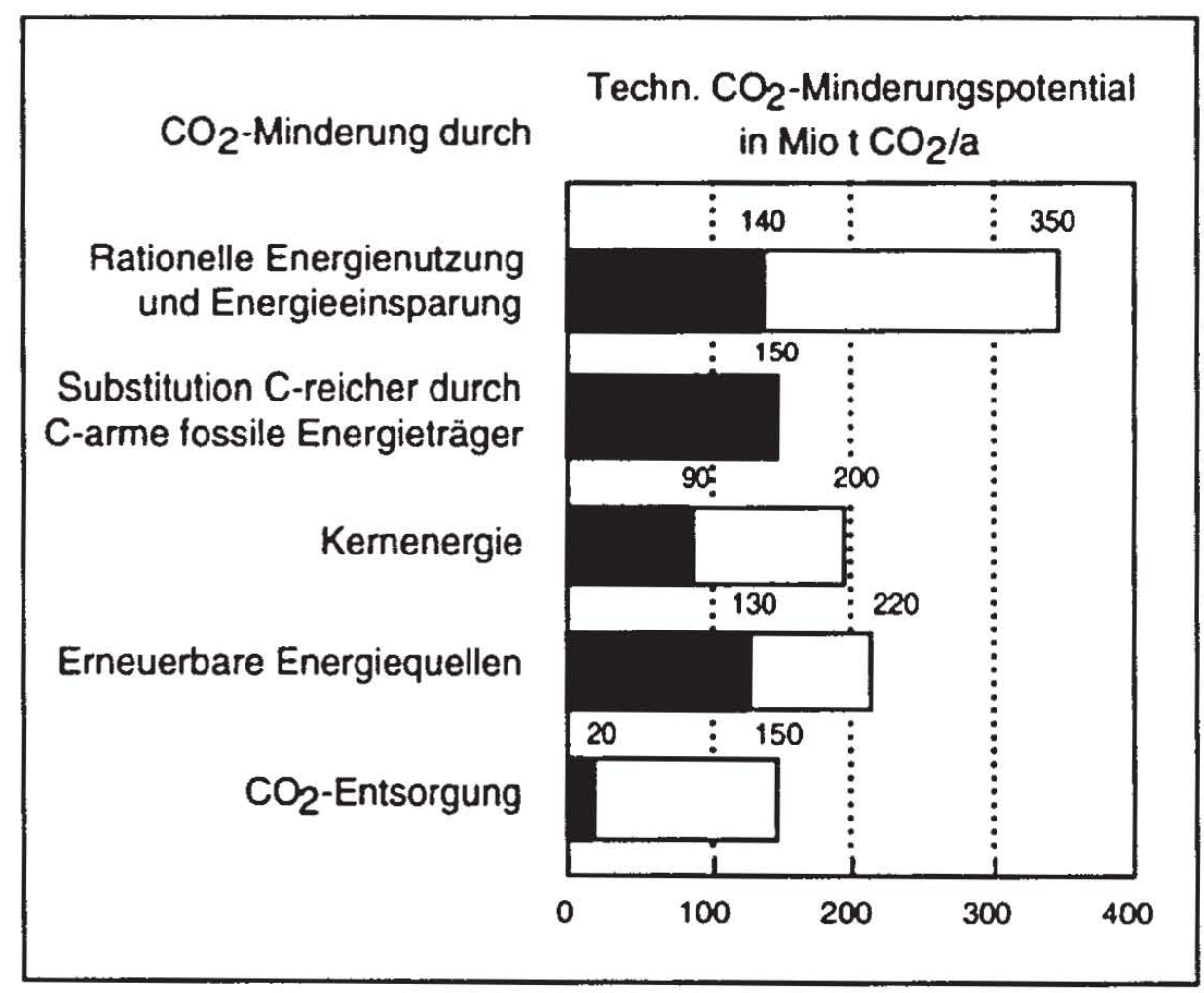

Bild 8 Technische $\mathrm{CO}_{2}$-Minderungspotentiale in der Bundesrepublik Deutschland bis zum Jahr 2005 
Das Vorhandensein nennenswerter technischer $\mathrm{CO}_{2}$-Minderungsmöglichkeiten sagt aber, wie anfangs bereits erläutert, noch nichts darüber aus, welche gesamtwirtschaftlichen Belastungen mit der Minderung von $\mathrm{CO}_{2}$ Emissionen verbunden sind, und welche $\mathrm{CO}_{2}$-Minderungsmöglichkeiten es erlauben, vorgegebene Minderungsziele mit den geringsten gesamtwirtschaftlichen Belastungen zu erreichen, oder wie die Aufwendungen für die $\mathrm{CO}_{2}$-Emissionsminderungen möglichst effizient genutzt, d. h. in maximale $\mathrm{CO}_{2}$-Minderungen umgesetzt werden.

\section{$\mathrm{CO}_{2}$-Reduktionsstrategien}

Im Rahmen der Arbeiten für die Enquête-Kommission Vorsorge zum Schutz der Erdatmosphäre sind, aufbauend auf den Einzelanalysen der verschiedenen $\mathrm{CO}_{2}$-Minderungsoptionen, erste Überlegungen bezüglich der Ausgestaltung von Strategien zur Verminderung energiebedingter $\mathrm{CO}_{2}$-Emissionen angestellt worden. Nach Vorgabe der Enquête-Kommission waren dabei drei Reduktionsszenarien für das Jahr $2005 \mathrm{zu}$ erarbeiten, die sich an dem Ziel einer etwa $30 \%$ igen $\mathrm{CO}_{2}$-Minderung (bezogen auf die Emissionen des Jahres 1987) orientieren und unterschiedliche energiepolitische Auffassungen reflektieren sollten. In einem ersten Szenario Hemmnisabbau und Preispolitik sollte der Energieeinsparung Priorität gegeben werden. Die Kernkraftwerkskapazität sollte auf dem gegenwärtigen Niveau eingefroren, aber höher ausgelastet werden und der Erdgaseinsatz sollte um nicht mehr als $30 \%$ zunehmen. In einem zweiten Szenario Kernenergieausstieg sollten die $\mathrm{CO}_{2}$-Minderungen unter der Annahme eines Verzichts auf die Nutzung der Kernenergie ab dem Jahr 2005 untersucht werden. Schließlich war ein drittes Szenario mit Ausbau der Kernenergie zu erstellen.

In Bild 9 ist der Versuch gemacht worden, wesentliche Ergebnisse der Reduktionsszenarien im Vergleich darzustellen. Die mit Trend bezeichnete Entwicklung der $\mathrm{CO}_{2}$-Emissionen beruht auf der Annahme, daß die gegenwärtigen Rahmenbedingungen der Energieversorgung im wesentlichen unverändert fortbestehen. Insbesondere werden keine speziellen Eingriffe zur Minderung der $\mathrm{CO}_{2}$-Emissionen unterstellt. Unter diesen Status-quo-Bedingungen bleiben die $\mathrm{CO}_{2}$-Emissionen bis zum Jahr 2005 nahezu unverändert auf dem Niveau des Jahres 1987.

In Anbetracht des im Jahr 2005 gut 50 \% höheren Bruttoinlandsproduktes und einer gestiegenen Energiedienstleistungsnachfrage bedeutet dies aber, daß die dem Trendszenario zugrundeliegenden Effizienzsteigerungen und Energieträgersubstitutionen bereits zu einer deutlichen Minderung des spezifischen $\mathrm{CO}_{2}$-Ausstosses geführt haben. Diese implizite 
$\mathrm{CO}_{2}$-Minderung, die ja aus heutiger Sicht auch noch zu leisten ist, läßt sich näherungsweise quantifizieren, wenn man die energetischen Nutzungsgrade und die Energieträgerstruktur des Jahres 1987 bis zum Jahr 2005 festschreibt. Unter dieser Annahme der frozen efficiency würde sich für das Jahr 2005 ein Anstieg der $\mathrm{CO}_{2}$-Emissionen auf rund 920 Mio. $t \mathrm{CO}_{2}$ ergeben. Die Differenz zu den $\mathrm{CO}_{2}$-Emissionen der Trendentwicklung in Höhe von 240 Mio. $\mathrm{CO}_{2}$ ist als Minderungsbedarf mit zu beachten, wenn man die angestrebte $\mathrm{CO}_{2}$-Minderung, d. h. die Minderungsziele, an dem $\mathrm{CO}_{2}$-Emissionsniveau des Jahres 1987 orientiert.

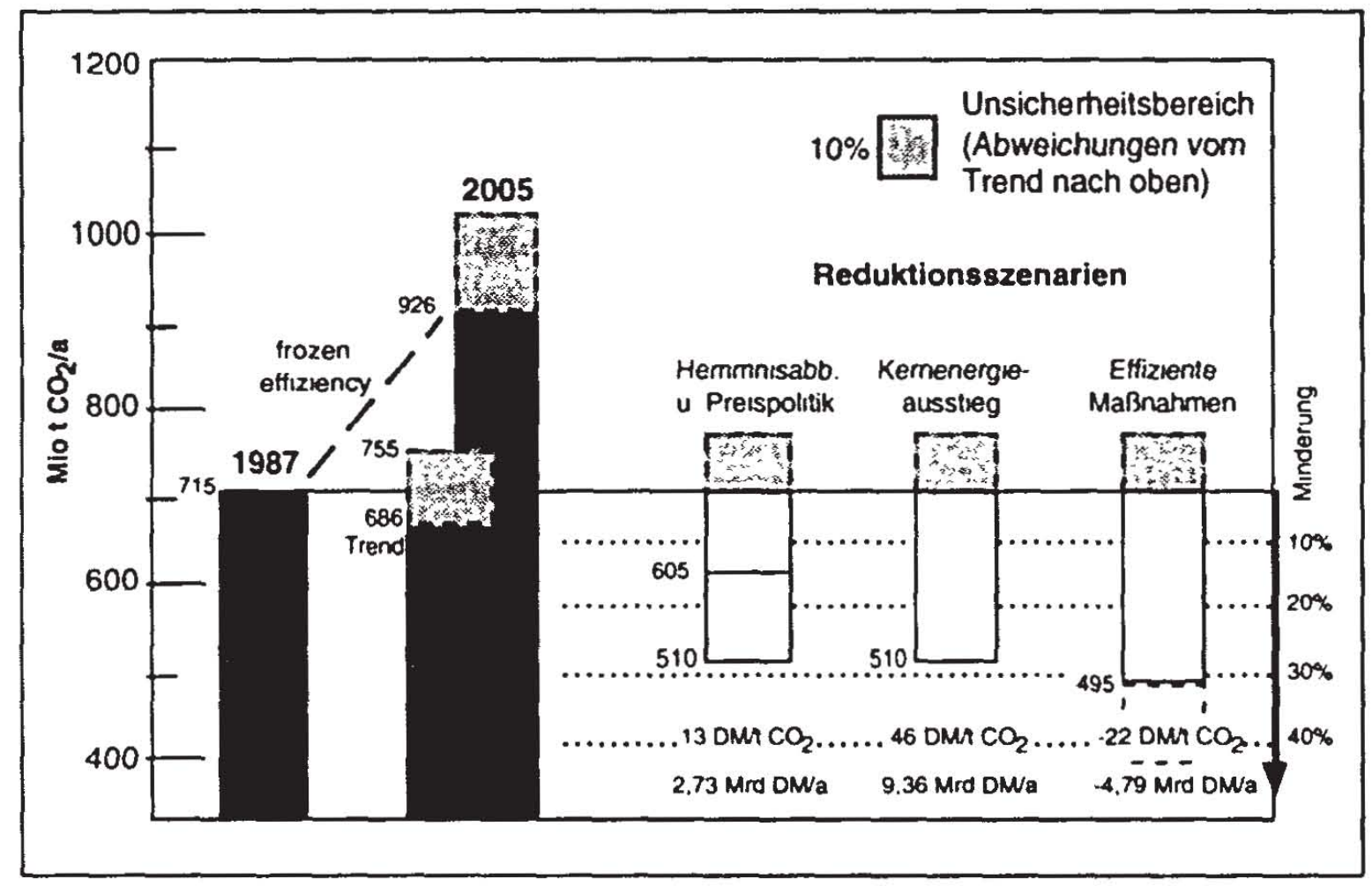

Bild $9 \quad \mathrm{CO}_{2}$-Reduktionsszenarien im Vergleich

Im Reduktionsszenario Hemmnisabbau und Preispolitik ist die $\mathrm{CO}_{2}$-Minderung zurückzuführen auf eine weitgehende Ausschöpfung der Einsparmöglichkeiten in allen Endverbraucherbereichen, eine Verlagerung und Reduktion von Verkehrsleistungen, eine erhebliche Ausweitung der Strom- und Wärmeerzeugung mittels erneuerbarer Energiequellen, nahezu eine Verdoppelung der KWK-Erzeugung und einen um $20 \% \mathrm{zu}$ nehmenden Erdgaseinsatz. Des weiteren werden $\mathrm{CO}_{2}$-Emissionen in Höhe von $27 \mathrm{Mio}$. $\mathrm{CO}_{2}$ durch eine bessere Auslastung der bestehenden Kernkraftwerke vermieden. 
Das Reduktionsszenario Kernenergieausstieg weist mit $510 \mathrm{Mio}$. $\mathrm{CO}_{2}$ die selben $\mathrm{CO}_{2}$-Emissionen und damit auch dieselben Emissionsreduktionen wie das Szenario Hemmnisabbau und Preispolitik aus. Um dies zu erreichen, wären aufgrund der Beendigung der Nutzung der Kernenergie im Jahr 2005 die Energieeinsparungen weiter zu verstärken, die Nutzung der erneuerbaren Energiequellen auszuweiten und der Erdgaseinsatz zu erhöhen. Die dazu notwendigen Maßnahmen seien an einigen Beispielen verdeutlicht. Für den Raumwärmebereich wird unterstellt, daß nahezu $40 \%$ des Altbaubestandes wärmetechnisch so saniert werden, daß der durchschnittliche Heizenergieverbrauch um zwei Drittel absinkt und alle Neubauten bis zum Jahr 2005 im Durchschnitt einen spez. Nettoheizenergiebedarf von $40 \mathrm{kWh} / \mathrm{m}^{2} \mathrm{a}$ bei Einfamilienhäusern bzw. $25 \mathrm{kWh} / \mathrm{m}^{2} \mathrm{a}$ bei Mehrfamilienhäusern aufweisen. Die Stromerzeugung in der Kraft-Wärme-Kopplung müßte etwa $2,8 \mathrm{mal}$ so hoch sein wie 1987. Die Stromerzeugungskapazität auf Basis erneuerbarer Energiequellen wäre bis 2005 um etwa 11,3 $\mathrm{GW}_{\mathrm{el}}$ auszuweiten, allein auf die Windkraft entfielen davon $5,2 \mathrm{GW}_{\mathrm{el}}$.

Im Reduktionsszenario mit Ausbau der Kernenergie sind die einzelnen $\mathrm{CO}_{2}$-Minderungsmaßnahmen weitgehend nach den Effizienzkriterien ausgewählt worden. Im Sinne einer effizienzorientierten $\mathrm{CO}_{2}$-Minderungsstrategie werden dabei alle im Rahmen der erwarteten Energiepreissteigerungen aus volkswirtschaftlicher Sicht sinnvollen Energieeinsparmöglichkeiten, auch durch eine verstärkte Kraft-Wärme-Kopplung, ebenso genutzt, wie die diesbezüglichen Potentiale der erneuerbaren Energiequellen. Durch den Ausbau der Kernenergie werden $\mathrm{CO}_{2}$-Emissionen in Höhe von $92 \mathrm{Mio}$. $\mathrm{CO}_{2} / \mathrm{a}$ vermieden. Dabei wird unterstellt, daß sich die installierte Bruttoengpaßleistung der Kernkraftwerke von heute 23,6 GW el auf $36,6 \mathrm{GW}_{\mathrm{el}}$ im Jahr 2005 erhöht. Insgesamt ergeben sich in diesem effizienzorientierten Reduktionsszenario mit Kernenergieausbau $\mathrm{CO}_{2}$ Emissionen im Jahr 2005 in Höhe von 495 Mio. t. Dies entspricht einer Minderung um 220 Mio. t $\mathrm{CO}_{2}$ oder $31 \%$ gegenüber dem Jahr 1987.

Vergleicht man die drei Reduktionsszenarien untereinander, so ergeben sich trotz der in der Größenordnung vergleichbaren $\mathrm{CO}_{2}$-Minderungen einige wesentliche Unterschiede. Sie liegen einmal in dem unterschiedlichen Kostenaufwand für die Erreichung der $\mathrm{CO}_{2}$-Minderung. Die jährlichen Nettokosten für die $\mathrm{CO}_{2}$-Minderungsmaßnahmen belaufen sich im Falle des Reduktionsszenarios Hemmnisabbau und Energiepolitik auf rund 2,7 Mrd. DM/a und im Fall des Reduktionsszenarios Kernenergieausstieg auf mehr als $9 \mathrm{Mrd}$. DM/a, wobei hier wegen fehlender Daten nicht alle Zusatzkosten erfaßt werden konnten und im Falle des Kernenergieausstiegs z. B. auch die Kapitalvernichtung durch die vorzeitige Stillegung der Kernkraftwerke nicht bewertet worden ist. Die Minderung 
der $\mathrm{CO}_{2}$-Emissionen im effizienzorientierten Reduktionsszenario mit Ausbau der Kernenergie wäre dagegen gegenüber der Trendentwicklung mit einer Kostenentlastung der Volkswirtschaft von rund 4,8 Mrd. DM/a verbunden. Im Vergleich zum Kernenergieausstiegsszenario ergäbe sich damit bei einer $\mathrm{CO}_{2}$-Minderung von rund $30 \%$ eine jährliche Kostendifferenz von mehr als 14 Mrd. DM.

Ein weiterer wesentlicher Unterschied zwischen den drei Reduktionsstrategien besteht im Hinblick auf ihre Möglichkeiten, falls notwendig, weitergehende $\mathrm{CO}_{2}$-Minderungsziele zu erreichen. Diese sind im Falle des Kernenergieausstiegs wohl nicht vorhanden. Würde man hingegen bei der Reduktionsstrategie mit Ausbau der Kernenergie, die in den beiden anderen Szenarien unterstellten weitergehenden Maßnahmen im Bereich der Energieeinsparung, der Ausweitung der Nutzung erneuerbarer Energiequellen und von Erdgas auch durchführen, so ließen sich die $\mathrm{CO}_{2}$-Emissionen um weitere $65 \mathrm{Mio}$. t/a reduzieren.

Obwohl die hier erläuterten $\mathrm{CO}_{2}$-Minderungsmöglichkeiten sich auf das Gebiet der alten Bundesländer beziehen, geben sie dennoch einen ersten Hinweis auf die in den neuen Bundesländern bestehenden Minderungspotentiale, wenn sich die Strukturen der Energieversorgung langfristig in eine ähnliche Richtung entwickeln.

Die hier erläuterten Ergebnisse von Untersuchungen zur Reduzierung der energiebedingten Treibhausgase sind nur als erste Phase einer Technikfolgenabschätzung zu betrachten und in vielerlei Hinsicht zu erweitern und abzusichern. Für einzelne Minderungsmaßnahmen liegen belastbare Kostenangaben und insbesondere Angaben zu Kosten in Gestalt von Zielverzichten nicht oder nur rudimentär vor. Eine umfassende Analyse und Bewertung der Vor- und Nachteile unterschiedlicher Treibhausgasminderungsstrategien, die auch die gesamtwirtschaftlichen Effekte, die umweltseitigen Vor- und Nachteile sowie die möglichen Preisrückwirkungen auf den internationalen Energiemärkten einbeziehen, ist noch zu leisten.

Dennoch lassen sich für den politischen Abwägungsproze $B$ erste Orientierungen gewinnen. Für die Bundesrepublik Deutschland (einschließlich der neuen Bundesländer auf die zuvor nicht eingegangen worden ist) existieren bereits mittelfristig, d. h. bis zum Jahr 2005, beachtliche technische Möglichkeiten zur Minderung der energiebedingten Treibhausgasemissionen. Ein Teil dieser Minderungspotentiale ließe sich ausschöpfen, ohne die Energiewirtschaft bzw. die Volkswirtschaft mit zusätzlichen Kosten zu belasten. Eine Ausnutzung dieser $\mathrm{CO}_{2}$-Minderungsmöglichkeiten, deren ökonomischer Nutzen allein schon größer ist als ihre Kosten, würde es der Bundesrepublik Deutschland zusammen mit den übri- 
gen Industrienationen erlauben, eine Schrittmacherrolle zu übernehmen, ohne die Volkswirtschaft einseitigen Belastungen auszusetzen.

\section{Schlußbemerkungen}

Aus den bisher vorliegenden TA-Studien im Energiebereich und den dabei gemachten Erfahrungen lassen sich u. a. folgende Schlußfolgerungen ableiten:

(1) Die großen Herausforderungen, denen wir uns an der Schwelle zum dritten Jahrtausend gegenübersehen, sind durch Komplexität und Vernetzung sowie eine immer stärkere internationale und globale Dimension gekennzeichnet. Die Energieprobleme und die mit ihr eng verknüpften Belastungen von Umwelt und Natur sowie die Gefahren einer Veränderung des Klimas sind angesichts einer weiter wachsenden Weltbevölkerung zentrale Aspekte dieser Weltproblematik. Die Erarbeitung tragfähiger Lösungen bzw. Lösungsansätze erfordert einen ganzheitlichen Ansatz und eine umfassende Analyse aller aus heutiger Kenntnis denkbaren Handlungsmöglichkeiten im Sinne einer möglichst genauen Quantifizierung ihrer im Zeitablauf möglichen Beiträge zur Bewältigung der Probleme, aber auch ihrer unerwünschten Nebeneffekte und Risiken.

(2) Technikfolgenabschätzung erfordert die Aufbereitung des vorhandenen Fachwissens aus verschiedensten Gebieten und die Einordnung dieses Fachwissens in den Systemzusammenhang. Zur Bearbeitung von TA-Studien sind daher interdisziplinäre Ansätze und interdisziplinäre Zusammenarbeit erforderlich.

(3) Nicht nur negative Folgen, sondern auch positive Auswirkungen des Technikeinsatzes sollten aufgezeigt werden, da nur so eine Gesamtwertung einer Technik möglich ist.

(4) Technikfolgenabschätzung ist zukunftsgerichtet - es geht um die Folgen von Technik und deren Veränderung in der Zukunft. Um die Wechselwirkung der Technik mit der Systemumgebung zu untersuchen, muß daher die zukünftige Systemumgebung (z. B. der zukünftige Strombedarf und die Deckung dieses Bedarfs) vorgegeben werden. Eine Prognose (im Sinne einer Vorhersage) ist aber nicht möglich. Es müssen daher mehrere Szenarien (Referenzszenarien) herangezogen werden, die die Bandbreite möglicher zukünftiger Entwicklungen darstellen.

(5) Zur Abbildung und Analyse des Energiesystems mit seinen vielfach vernetzten Strukturen im Rahmen von Technikfolgenabschätzungen sind 
Energiemodelle (Simulations- und Optimierungsmodelle) ein unverzichtbares Hilfsmittel.

(6) Nicht alle Folgen der Energieumwandlung lassen sich heute bereits befriedigend quantifizieren. So ist $\mathrm{z}$. B. noch nicht endgültig geklärt, auf welche Weise und in welchem Ausmaß Luftschadstoffe zu den neuartigen Waldschäden beitragen. Auch der Zusammenhang zwischen Luftschadstoffkonzentrationen und Atemwegserkrankungen wird noch kontrovers diskutiert. In solchen Fällen sind die Abschätzungen von Technikfolgen noch mit Lücken oder Unsicherheiten behaftet, Technikfolgenforschung ist erforderlich.

(7) Technikfolgenabschätzung wird nur dann eine brauchbare Grundlage für den notwendigen Diskurs zur Technikbewertung sein können, wenn sie der Erarbeitung von Fakten und der wissenschaftlichen Analyse breiten Raum gibt und die Bearbeiter sich eigener Wertungen so weit wie möglich enthalten.

(8) In der Energiepolitik und die Energiewirtschaft müssen derzeit und in Zukunft Entscheidungen mit großer Tragweite gefällt werden. Eine systematische Weiterentwicklung von Instrumenten zur Technikfolgenabschätzung im Energiebereich und die Anwendung dieser Instrumente scheint dringend geboten.

Abschließend sei angemerkt, daß die bisher für den Energiebereich durchgeführten probleminduzierten Technikfolgenabschätzungen auch gezeigt haben, daß, wie Carl Friedrich von Weizsäcker es einmal ausgedrückt hat, "alle Gefahren, die wir vor uns sehen, keine technischen Ausweglosigkeiten sind, sondern eher umgekehrt, die Unfähigkeit unserer Kultur, mit den Geschenken ihrer eigenen Erfindungskraft vernünftig umzugehen."

\section{$5 \quad$ Literaturverzeichnis}

/1/ Bundesministerium für Wirtschaft (Hrsg.): Energiedaten 1991, Bonn, Sept. 1992.

12/ Dritter Bericht der Enquête-Kommission Vorsorge zum Schutz der Erdatmosphäre, Bundestagsdrucksache 11/8030, Bonn, 24.5.1990.

13/ Statistisches Bundesamt (Hrsg.): Statistisches Jahrbuch 1990, Stuttgart 1990.

14/ Umweltbundesamt (Hrsg.): Daten zur Umwelt 1988/89, Berlin 1989. 
15/ Bericht der Enquête-Kommission "Gestaltung der technischen Entwicklung; Technikfolgenabschätzung und Bewertung": Bedingungen und Folgen von Aufbaustrategien für eine solare Wasserstoffwirtschaft; Bundestagsdrucksache 11/7993, Bonn 24.9.90.

/6/ A. Voß (Hrsg.): Perspektiven der Energieversorgung Baden-Württembergs unter besonderer Berücksichtigung der Stromversorgung, Stuttgart November 1987.

17/ Umweltbundesamt (Hrsg.): Daten zur Umwelt 1990/91, Berlin 1992.

18/ Bundesminister für Verkehr (Hrsg.): Verkehr in Zahlen 1993, Berlin 1993. 